\title{
Analytical solutions of compacting flow past a sphere
}

\author{
John F. Rudge $\dagger$ \\ Bullard Laboratories, Department of Earth Sciences, University of Cambridge, Madingley \\ Road, Cambridge CB3 0EZ, UK
}

(Received ?; revised ?; accepted ?. - To be entered by editorial office)

A series of analytical solutions are presented for viscous compacting flow past a rigid impermeable sphere. The sphere is surrounded by a two-phase medium consisting of a viscously deformable solid matrix skeleton through which a low viscosity liquid melt can percolate. The flow of the two-phase medium is described by McKenzie's compaction equations which combine Darcy flow of the liquid melt with Stokes flow of the solid matrix. The analytical solutions are found using an extension of the Papkovich-Neuber technique for Stokes flow. Solutions are presented for the three components of linear flow past a sphere: translation, rotation, and straining flow. Faxén laws for the force, torque, and stresslet on a rigid sphere in an arbitrary compacting flow are derived. The analytical solutions provide instantaneous solutions to the compaction equations in a uniform medium, but can also be used to numerically calculate an approximate evolution of the porosity over time whilst the porosity variations remain small. These solutions will be useful for interpreting the results of deformation experiments on partially molten rocks.

\section{Introduction}

On geological time scales Earth's solid mantle flows and convects, the well-known surface expression of this being plate tectonics. At mid-ocean ridges two plates separate, causing solid mantle material to upwell, decompress, and partially melt. The melt rises to the surface to form new oceanic crust. Despite several decades of work, it still not clear exactly how melt is transported to the surface from where it is first formed at depths of around $100 \mathrm{~km}$ within the Earth. Understanding the transport of melt in the mantle is a formidable fluid dynamical challenge, as it requires an understanding of the interaction between two phases with very different properties: the solid mantle (with a viscosity around $10^{21} \mathrm{~Pa} \mathrm{~s}$ ) and the liquid melt (with a viscosity around $1 \mathrm{~Pa} \mathrm{~s}$ ), and a understanding of physics on a wide range of scales: from the grain scale to plate-tectonic scales.

The two-phase flow of melt in the Earth's mantle has been extensively modelled using McKenzie's compaction equations (McKenzie 1984). These equations describe a viscously deformable solid matrix skeleton through which a low viscosity melt phase can percolate. While it is not yet clear how good a model the compaction equations are for the transport of melt in the Earth's mantle, the use of these equations has now become standard (e.g. Hewitt \& Fowler (2009); Katz (2010); Mittelstaedt et al. (2011); Schiemenz et al. (2011)). One way of testing the validity of the compaction equations as a model for melt transport is to examine the deformation behaviour of partially molten rocks in the laboratory (Kohlstedt \& Holtzman 2009), comparing the results of physical experiments

$\dagger$ Email address for correspondence: rudge@esc.cam.ac.uk 
to solutions of the compaction equations (e.g. Katz et al. (2006)). The hope is that through such comparisons, insights can be gained into the applicability of the compaction equations in describing the deformation of partially molten rocks, and moreover that the experiments will place constraints on the appropriate numerical values of parameters in the compaction equations, such as the shear viscosity, bulk viscosity, and permeability.

The compaction equations describe a generalisation of Stokes flow to add percolation of melt to the viscous deformation of the solid matrix. One of the classic problems in the theory of Stokes flow is that of uniform flow past a rigid sphere. Indeed, this problem was solved by Stokes himself, and can be found in most elementary textbooks on fluid dynamics (e.g. Acheson (1990); Batchelor (1967)). The aim of this manuscript is to study compacting flow past a sphere by finding the corresponding solutions of McKenzie's compaction equations. Unlike the Stokes flow equations, there is a natural lengthscale in the compaction equations termed the compaction length. Explicit solutions to compacting flow past a sphere have already been found by McKenzie \& Holness (2000) in the particular limit of large compaction length. The present study extends the work of McKenzie \& Holness (2000) in three main ways: First, explicit solutions are obtained for arbitrary compaction length. Second, expressions are derived for the force, torque, and stresslet exerted on a rigid sphere by an arbitrary compacting flow. Third, the expected time evolution of porosity is calculated.

The present manuscript is motivated by ongoing experiments designed to study the deformation of a partially molten material containing rigid spherical inclusions (Qi et al. 2013). In these experiments a partially molten fine-grained $(10 \mu \mathrm{m})$ aggregate of San Carlos olivine plus mid-ocean ridge basalt is deformed in torsion. Within the aggregate are spherical single crystal beads of olivine, whose size is much smaller than the overall sample size ( $0.35 \mathrm{~mm}$ radii spheres and $12 \mathrm{~mm}$ diameter samples). The beads undergo little deformation, and are effectively rigid. The flow field in the neighbourhood of the beads is effectively simple shear due to the small size of the beads compared with the sample size. The role of this manuscript is to provide the analytical solutions necessary to interpret the results from these experiments, by providing solutions to compacting flow past a rigid sphere in simple shear. Future manuscripts will compare the theoretical predictions with the experimental results, and will also develop numerical solutions to the full non-linear set of equations. The present manuscript presents a generalisation of the well-known analytical results for Stokes flow past a sphere, similar to the treatment given in Chapter 2 of the textbook by Guazzelli \& Morris (2012).

The manuscript is organised as follows: The next section presents the compaction equations, and is followed by a section describing decompositions of the compaction equations in terms of potentials. Spherically symmetric solutions of the compaction equations are then discussed, before moving on to the main results: the solutions to compacting flow past a rigid sphere. Expressions for the force, torque, and stresslet are then obtained, and are followed by a discussion of the porosity evolution. An appendix describes a related solution for compacting flow past a circular cylinder.

\section{The compaction equations}

The two phase flow of melt and matrix is described by the following set of equations (McKenzie 1984), where it is assumed that all body forces can be neglected, that there 
is no melting, and that the density of the two phases are equal and constant:

$$
\begin{gathered}
-\frac{\partial \phi}{\partial t}+\boldsymbol{\nabla} \cdot\left((1-\phi) \boldsymbol{v}_{s}\right)=0, \\
\boldsymbol{\nabla} \cdot\left(\boldsymbol{v}_{s}+\boldsymbol{q}\right)=0, \\
\boldsymbol{q}=-\frac{\mathrm{k}_{\phi}}{\mu} \boldsymbol{\nabla} P \\
\boldsymbol{\nabla} \cdot \bar{\sigma}=\mathbf{0} \\
\bar{\sigma}=-P \boldsymbol{I}+\zeta_{\phi}\left(\boldsymbol{\nabla} \cdot \boldsymbol{v}_{s}\right) I+\eta_{\phi}\left(\boldsymbol{\nabla} \boldsymbol{v}_{s}+\boldsymbol{\nabla} \boldsymbol{v}_{s}^{T}-\frac{2}{3}\left(\boldsymbol{\nabla} \cdot \boldsymbol{v}_{s}\right) l\right) .
\end{gathered}
$$

(2.1) is conservation of mass for the solid matrix where $\phi$ is the porosity and $\boldsymbol{v}_{s}$ is the matrix velocity. (2.2) is conservation of mass for the overall two phase mixture where $\boldsymbol{q} \equiv \phi\left(\boldsymbol{v}_{f}-\boldsymbol{v}_{s}\right)$ is the Darcy flux, and $\boldsymbol{v}_{f}$ is the melt velocity. (2.3) is conservation of momentum for the melt, Darcy's law, where $k_{\phi}$ is the permeability and $\mu$ is the melt viscosity. (2.4) is conservation of momentum for the overall two phase mixture where $\bar{\sigma}$ is the mean stress tensor. (2.5) provides a constitutive law for the mean stress tensor, where $P$ is the melt pressure, $\eta_{\phi}$ is the effective shear viscosity and $\zeta_{\phi}$ the effective bulk viscosity of the two phase mixture. Subscripts $\phi$ on $\mathrm{k}_{\phi}, \eta_{\phi}$ and $\zeta_{\phi}$ indicate that these properties depend on porosity, and to complete the set of governing equations additional constitutive laws are required for these properties. Further detailed discussion of the origin of these equations can be found in McKenzie (1984); Ricard (2007); Rudge et al. (2011); Simpson et al. (2010); Spiegelman (1993).

In this work we are primarily concerned with the instantaneous compaction around the sphere. We thus defer discussion of the time evolution of porosity given by (2.1) to section 7 . The remaining equations $(2.2-2.5)$ can be combined to give

$$
\begin{gathered}
\boldsymbol{\nabla} \cdot \boldsymbol{v}_{s}-\boldsymbol{\nabla} \cdot\left(\frac{\mathrm{k}_{\phi}}{\mu} \boldsymbol{\nabla} P\right)=0, \\
-\boldsymbol{\nabla} P+\boldsymbol{\nabla}\left(\left(\zeta_{\phi}-\frac{2}{3} \eta_{\phi}\right) \boldsymbol{\nabla} \cdot \boldsymbol{v}_{s}\right)+\boldsymbol{\nabla} \cdot\left(\eta_{\phi}\left(\boldsymbol{\nabla} \boldsymbol{v}_{s}+\boldsymbol{\nabla} \boldsymbol{v}_{s}^{T}\right)\right)=\mathbf{0} .
\end{gathered}
$$

Given a porosity field $\phi,(2.6)$ and (2.7) can be solved to find the matrix velocity $\boldsymbol{v}_{\boldsymbol{s}}$ and the melt pressure $P$. The corresponding Darcy flux can then be obtained from (2.3).

We will assume that the medium is uniform, i.e. that $\mathrm{k}_{\phi}, \eta_{\phi}$, and $\zeta_{\phi}$ are uniform and so drop the $\phi$ subscripts. We will non-dimensionalise on the natural length scale in the problem, the compaction length $\delta$, defined by (McKenzie 1984)

$$
\delta=\sqrt{\frac{\mathrm{k}\left(\zeta+\frac{4}{3} \eta\right)}{\mu}} .
$$

The non-dimensional scalings are

$$
\boldsymbol{x}=\delta \boldsymbol{x}^{\prime}, \quad \boldsymbol{v}_{s}=\frac{\delta}{\tau} \boldsymbol{v}_{s}^{\prime}, \quad P=\frac{\zeta+\frac{4}{3} \eta}{\tau} P^{\prime},
$$

where $\tau$ is an arbitrary time scale. (2.6) and (2.7) can then be written in dimensionless form as

$$
\begin{gathered}
\boldsymbol{\nabla} \cdot \boldsymbol{v}_{s}-\nabla^{2} P=0, \\
-\boldsymbol{\nabla} P+\boldsymbol{\nabla}\left(\boldsymbol{\nabla} \cdot \boldsymbol{v}_{s}\right)-\mathcal{B} \boldsymbol{\nabla} \times\left(\boldsymbol{\nabla} \times \boldsymbol{v}_{s}\right)=\mathbf{0},
\end{gathered}
$$


where

$$
\mathcal{B}=\frac{\eta}{\zeta+\frac{4}{3} \eta}=\frac{1}{R+\frac{4}{3}},
$$

and $R=\zeta / \eta$ is the ratio of the bulk viscosity to the shear viscosity. Note that $0 \leq \mathcal{B} \leq$ $3 / 4$.

\subsection{Limiting cases}

There are two important limiting cases of (2.10) and (2.11) depending on whether the radius of the sphere $r$ is small or large compared with the compaction length $\delta$ (McKenzie 1984; McKenzie \& Holness 2000). For $a \equiv \mathrm{r} / \delta \ll 1$ (large compaction length) (2.10) and (2.11) can be approximated in the neighbourhood of the sphere as

$$
\begin{gathered}
\boldsymbol{\nabla} \cdot \boldsymbol{v}_{s}-\nabla^{2} P=0, \\
(1-\mathcal{B}) \boldsymbol{\nabla}\left(\boldsymbol{\nabla} \cdot \boldsymbol{v}_{s}\right)+\mathcal{B} \nabla^{2} \boldsymbol{v}_{s} \approx \mathbf{0},
\end{gathered}
$$

where (2.14) can be recognised as the Navier-Cauchy equation of elastostatics. Notice that the two equations are decoupled: (2.14) can be solved to obtain the matrix velocity $\boldsymbol{v}_{s}$, which can then be used in (2.13) to find the fluid pressure $P$. Solutions of (2.14) around spheres are well known and can be found in textbooks such as Phan-Thien \& Kim (1994). This is the limit in which the explicit solutions of McKenzie \& Holness (2000) were found, and indeed the solutions they found for the matrix velocity $\boldsymbol{v}_{s}$ are identical to the solutions for elastic inclusions presented in Phan-Thien \& Kim (1994).

If $a \gg 1$ (small compaction length) (2.10) and (2.11) can be approximated as

$$
\begin{gathered}
\boldsymbol{\nabla} \cdot \boldsymbol{v}_{s} \approx 0, \\
-\boldsymbol{\nabla} P+\mathcal{B} \nabla^{2} \boldsymbol{v}_{s} \approx \mathbf{0}
\end{gathered}
$$

i.e. the equations of incompressible Stokes flow. Formally, the limit $a \rightarrow \infty$ is singular, where the compaction length $\delta$ controls the thickness of the corresponding boundary layers at the sphere surface. Solutions to Stokes flows around spheres are very well known, and can be found in textbooks such as Guazzelli \& Morris (2012); Kim \& Karrila (2005).

It is an important check on the full solutions of (2.10) and (2.11) that they recover the Stokes and Navier-Cauchy solutions in the two limits described above.

\section{Potential form}

A convenient way of writing the solutions to (2.10) and (2.11) is in terms of potentials. This has been exploited to good effect by previous authors using a Helmholtz decomposition (Spiegelman 1993). We recall this decomposition here, and also introduce a new decomposition based upon Papkovich-Neuber potentials.

\subsection{Helmholtz decomposition}

Key to both decompositions is the fact that the diverging (compacting) part of the flow $\boldsymbol{v}_{s}$ can be separated from the divergence-free (non-compacting) part in a uniform medium. We define

$$
\mathcal{C}=\nabla \cdot v_{s}
$$

to be the compaction rate and

$$
\boldsymbol{\omega}_{s}=\boldsymbol{\nabla} \times \boldsymbol{v}_{s}
$$


to be the solid vorticity. By the Helmholtz theorem (e.g. Appendix B, Griffiths (1999)), knowledge of $\mathcal{C}$ and $\boldsymbol{\omega}_{s}$ is sufficient to be able to recover $\boldsymbol{v}_{s}$ (assuming an appropriate decay of these fields at infinity).

Taking the divergence of (2.11) and substituting it into (2.10) yields an equation for the compaction rate

$$
-\nabla^{2} \mathcal{C}+\mathcal{C}=0
$$

the homogeneous modified Helmholtz equation.

Taking the curl of (2.11) yields

$$
\nabla^{2} \boldsymbol{\omega}_{s}=\mathbf{0}
$$

and thus the solid vorticity is harmonic.

Using a Helmholtz decomposition the solid velocity field is written as

$$
\boldsymbol{v}_{s}=\boldsymbol{\nabla U}+\boldsymbol{\nabla} \times \boldsymbol{\Psi}
$$

in terms of a scalar potential $\mathcal{U}$ and a vector potential $\boldsymbol{\Psi}$. Without loss of generality we may assume $\boldsymbol{\nabla} \cdot \boldsymbol{\Psi}=0$ (a gauge condition). Taking the divergence and the curl of (3.5) then yields

$$
\begin{gathered}
\mathcal{C}=\nabla^{2} \mathcal{U}, \\
\boldsymbol{\omega}_{s}=-\nabla^{2} \boldsymbol{\Psi} .
\end{gathered}
$$

The pair of equations (3.3) and (3.6) can be solved for the scalar potential $\mathcal{U}$, and the pair of equations (3.4) and (3.7) for the vector potential $\boldsymbol{\Psi}$. It follows from (3.4) and (3.7) that $\nabla^{4} \boldsymbol{\Psi}=\mathbf{0}$, i.e. the vector potential $\boldsymbol{\Psi}$ satisfies the biharmonic equation.

\subsection{Papkovich-Neuber potentials}

The second term of the right-hand side of (3.5), $\boldsymbol{\nabla} \times \boldsymbol{\Psi}$, is a constant viscosity Stokes flow. There are a wide variety of different representations of Stokes flow, one of the more useful ones being the Papkovich-Neuber representation (Lister et al. 2011; Neuber 1934; Papkovich 1932; Phan-Thien \& Kim 1994). This representation writes the Stokes flow as

$$
\boldsymbol{\nabla} \times \boldsymbol{\Psi}=\boldsymbol{\nabla}(\boldsymbol{x} \cdot \boldsymbol{\varphi}+\theta)-2 \boldsymbol{\varphi},
$$

where $\varphi$ and $\theta$ are harmonic potentials, $\nabla^{2} \boldsymbol{\varphi}=\mathbf{0}, \nabla^{2} \theta=0$, and $\boldsymbol{x}$ is the position vector. This representation enables Stokes flow solutions to be easily constructed from solutions of Laplace's equation.

From (3.3) and (3.6) it follows that $\mathcal{V} \equiv \mathcal{U}-\mathcal{C}$ is harmonic, $\nabla^{2} \mathcal{V}=0$. Hence the compacting part of the flow may be written as

$$
\nabla \mathcal{U}=\nabla \mathcal{C}+\nabla \mathcal{V}
$$

where $\nabla^{2} \mathcal{C}=\mathcal{C}$ and $\nabla^{2} \mathcal{V}=0$. Thus solutions of the compacting part of the flow can be constructed from solutions of the modified Helmholtz equation and Laplace's equation.

Combining (3.5), (3.8), and (3.9) yields a general decomposition of the solid velocity field $\boldsymbol{v}_{s}$ as

$$
\boldsymbol{v}_{s}=\boldsymbol{\nabla}(\boldsymbol{x} \cdot \boldsymbol{\varphi}+\chi+\mathcal{C})-2 \boldsymbol{\varphi},
$$

where $\varphi$ and $\chi$ are harmonic $\nabla^{2} \boldsymbol{\varphi}=\mathbf{0}, \nabla^{2} \chi=0$, and $\mathcal{C}$ satisfies the modified Helmholtz equation $\nabla^{2} \mathcal{C}=\mathcal{C}$. Note that the two scalar harmonic potentials $\theta$ and $\mathcal{V}$ in (3.8) and (3.9) have been been combined to give a single scalar harmonic potential $\chi=\theta+\mathcal{V}$. (3.10) is the decomposition that will be exploited throughout this manuscript, where solutions of the compaction equations will be constructed from elementary solutions of Laplace's equation and the modified Helmholtz equation. 
By substituting (3.10) into the original compaction equations it can be seen that other quantities of interest have expressions in terms of these potentials as

$$
\begin{gathered}
P=2 \mathcal{B} \nabla \cdot \varphi+\mathcal{C}, \\
\boldsymbol{q}=-2 \mathcal{B} \nabla(\boldsymbol{\nabla} \cdot \boldsymbol{\varphi})-\boldsymbol{\nabla C}, \\
\boldsymbol{\omega}_{s}=-2 \boldsymbol{\nabla} \times \boldsymbol{\varphi}, \\
e \equiv \frac{1}{2}\left(\boldsymbol{\nabla} \boldsymbol{v}_{s}+\boldsymbol{\nabla} \boldsymbol{v}_{s}^{T}\right)=(\boldsymbol{\nabla} \boldsymbol{\nabla}) \cdot \boldsymbol{x}+\boldsymbol{\nabla} \boldsymbol{\nabla}(\chi+\mathcal{C}), \\
\bar{\sigma}=2 \mathcal{B}(-(\boldsymbol{\nabla} \cdot \boldsymbol{\varphi}+\mathcal{C}) \boldsymbol{I}+e),
\end{gathered}
$$

where $e$ is the rate of strain tensor.

\subsection{Fundamental solutions}

It is useful to recall the fundamental solutions of Laplace's equation and the modified Helmholtz equation. The fundamental solution of Laplace's equation is defined as the solution of

$$
-\nabla^{2} \chi=\delta^{(3)}(\boldsymbol{x})
$$

where $\delta^{(3)}(\boldsymbol{x})$ is the three-dimensional Dirac delta function. The fundamental solution is

$$
\chi=\frac{1}{4 \pi r}
$$

where $r=|\boldsymbol{x}|$. This fundamental solution satisfies $\nabla^{2} \chi=0$ for $r>0$, and decays to zero as $r \rightarrow \infty$. Importantly, additional solutions to Laplace's equation can be constructed simply by taking the gradient of this fundamental solution: $\nabla \chi, \nabla \nabla \chi, \nabla \nabla \nabla \chi$, etc are also harmonic functions. For example, the first two of these gradients are given by

$$
\begin{gathered}
\nabla\left(\frac{1}{r}\right)=-\frac{\boldsymbol{x}}{r^{3}}, \\
\nabla \nabla\left(\frac{1}{r}\right)=-\frac{l}{r^{3}}+\frac{3 \boldsymbol{x} \boldsymbol{x}}{r^{5}} .
\end{gathered}
$$

The set of these gradients are termed the spherical solid harmonics (Batchelor 1967), and form the basis for multipole expansions.

The fundamental solution of the modified Helmholtz equation satisfies

$$
-\nabla^{2} \mathcal{C}+\mathcal{C}=\delta^{(3)}(\boldsymbol{x})
$$

and is given by

$$
\mathcal{C}=\frac{k_{0}(r)}{4 \pi}
$$

where

$$
k_{0}(r) \equiv \frac{\mathrm{e}^{-r}}{r}
$$

is the zeroth order modified spherical Bessel function of the second kind. Additional solutions of the modified Helmholtz equation can also be generated by taking gradients. The spherical Bessel functions satisfy the following recursion relationship

$$
k_{n+1}(r)=-r^{n} \frac{\mathrm{d}}{\mathrm{d} r}\left(\frac{k_{n}(r)}{r^{n}}\right)
$$


so that

$$
\begin{gathered}
\boldsymbol{\nabla} k_{0}(r)=-\frac{k_{1}(r)}{r} \boldsymbol{x} \\
\nabla \nabla k_{0}(r)=-\frac{k_{1}(r)}{r} l+\frac{k_{2}(r) \boldsymbol{x} \boldsymbol{x}}{r^{2}}
\end{gathered}
$$

where

$$
\begin{gathered}
k_{1}(r)=\frac{\mathrm{e}^{-r}}{r^{2}}(1+r), \\
k_{2}(r)=\frac{\mathrm{e}^{-r}}{r^{3}}\left(3+3 r+r^{2}\right) .
\end{gathered}
$$

The next two sections present a series of analytical solutions to compacting flow around a sphere. The method for finding the solutions is the same in each case: educated guesses are made at the appropriate forms for the Papkovich-Neuber potentials which are then substituted into (3.10) to find the flow field. Boundary conditions are applied that determine the unknown constants. Since the solutions of the compaction equations are unique under suitable boundary conditions (Appendix B), once the boundary conditions have been satisfied it is known that the unique solution of the problem has been found.

\section{Spherically symmetric solutions}

It is instructive to begin by considering the simplest solutions to the compaction equations in 3D: the spherically symmetric solutions. These can be formed by using the fundamental solutions (3.17) and (3.21) as the scalar Papkovich-Neuber potentials,

$$
\chi=\frac{A}{r}, \quad \mathcal{C}=B k_{0}(r),
$$

for unknown constants $A$ and $B$. Substitution into (3.10-3.15) leads to

$$
\begin{gathered}
\boldsymbol{v}_{s}=-\left(\frac{A}{r^{2}}+B k_{1}(r)\right) \frac{\boldsymbol{x}}{r} \\
P=B k_{0}(r), \\
\boldsymbol{q}=B k_{1}(r) \frac{\boldsymbol{x}}{r} \\
\boldsymbol{\omega}_{s}=\mathbf{0} \\
\bar{\sigma}=2 \mathcal{B}\left[A\left(-\frac{I}{r^{3}}+\frac{3 \boldsymbol{x} \boldsymbol{x}}{r^{5}}\right)+B\left(-\left(k_{0}(r)+\frac{k_{1}(r)}{r}\right) I+\frac{k_{2}(r) \boldsymbol{x} \boldsymbol{x}}{r^{2}}\right)\right], \\
\bar{\sigma} \cdot \frac{\boldsymbol{x}}{r}=4 \mathcal{B}\left(\frac{A}{r^{2}}+B k_{1}(r)\right) \frac{\boldsymbol{x}}{r^{2}} .
\end{gathered}
$$

\subsection{Uniform expansion}

One example of a spherically symmetric problem is the case of a uniform expanding impermeable sphere. This has boundary conditions on the sphere

$$
\left.\boldsymbol{v}_{s}\right|_{r=a}=\frac{Q \boldsymbol{x}}{4 \pi a^{3}},\left.\quad \boldsymbol{q} \cdot \boldsymbol{n}\right|_{r=a}=0
$$

where $Q$ gives the total mass flux over the surface $r=a$ and $\boldsymbol{n}$ is the outward normal. It is assumed that $\boldsymbol{v}_{s} \rightarrow 0$ and $P \rightarrow 0$ as $|\boldsymbol{x}| \rightarrow \infty$. This has solution

$$
A=-\frac{Q}{4 \pi}, \quad B=0,
$$


so that

$$
\boldsymbol{v}_{s}=\frac{Q \boldsymbol{x}}{4 \pi r^{3}}, \quad \boldsymbol{q}=\mathbf{0}, \quad P=0,
$$

and the flow $\boldsymbol{v}_{s}$ of the matrix is simply that of a standard point source in Stokes flow.

\subsection{Melt injection}

A more interesting spherically symmetric example concerns a sphere of pure melt with a point source at its centre, representing an injection of melt. This example involves compaction, and highlights the difference between the compaction equations and the ordinary Stokes flow equations. At the surface of the melt sphere, mass must be conserved which gives that

$$
\overline{\boldsymbol{v}}=\boldsymbol{v}_{s}+\boldsymbol{q}=\frac{Q \boldsymbol{x}}{4 \pi a^{3}} \text { on } r=a,
$$

where $Q$ is the strength of the melt source. Assuming the melt pressure is continuous between the two-phase and single-phase regions, continuity of traction implies

$$
\bar{\sigma} \cdot \boldsymbol{n}=-P \boldsymbol{n} \text { on } r=a .
$$

These yield

$$
A=-\frac{Q}{4 \pi}, \quad B=\frac{Q}{4 \pi a^{2}\left(k_{1}(a)+a k_{0}(a) / 4 \mathcal{B}\right)} .
$$

\subsubsection{Point injection of melt}

In the limit that $a \rightarrow 0,(4.13)$ becomes

$$
A=-\frac{Q}{4 \pi}, \quad B=\frac{Q}{4 \pi},
$$

and the solution is that of a point injection of melt into a two phase medium,

$$
\begin{gathered}
\boldsymbol{v}_{s}=\frac{Q}{4 \pi}\left(\frac{1}{r^{2}}-k_{1}(r)\right) \frac{\boldsymbol{x}}{r}, \\
\boldsymbol{q}=\frac{Q}{4 \pi} k_{1}(r) \frac{\boldsymbol{x}}{r}, \\
P=\frac{Q}{4 \pi} k_{0}(r) .
\end{gathered}
$$

Formally, this is the solution of

$$
-\nabla^{2} P+\nabla \cdot \boldsymbol{v}_{s}=Q \delta^{(3)}(\boldsymbol{x}), \quad \boldsymbol{\nabla} \cdot \bar{\sigma}=0 .
$$

(4.15-4.17) represents one of the simplest examples of a solution to the compaction equations in 3D that is not a Stokes flow, and the radial components of this flow are plotted in figure 1. The point injection of melt causes compaction around the point over a lengthscale on the order of the compaction length. Far from the point of injection, the flow resembles that of an ordinary point source, since for $r \gg 1$,

$$
\boldsymbol{v}_{s} \sim \frac{Q \boldsymbol{x}}{4 \pi r^{3}}, \quad \boldsymbol{q} \sim \mathbf{0},
$$

and no compaction takes place in the far-field. Near the point of injection, $r \ll 1$,

$$
\boldsymbol{v}_{s} \sim \frac{Q \boldsymbol{x}}{8 \pi r}, \quad \boldsymbol{q} \sim \frac{Q \boldsymbol{x}}{4 \pi r^{3}},
$$

and compaction takes place, with the melt rapidly percolating away from the injection point. 


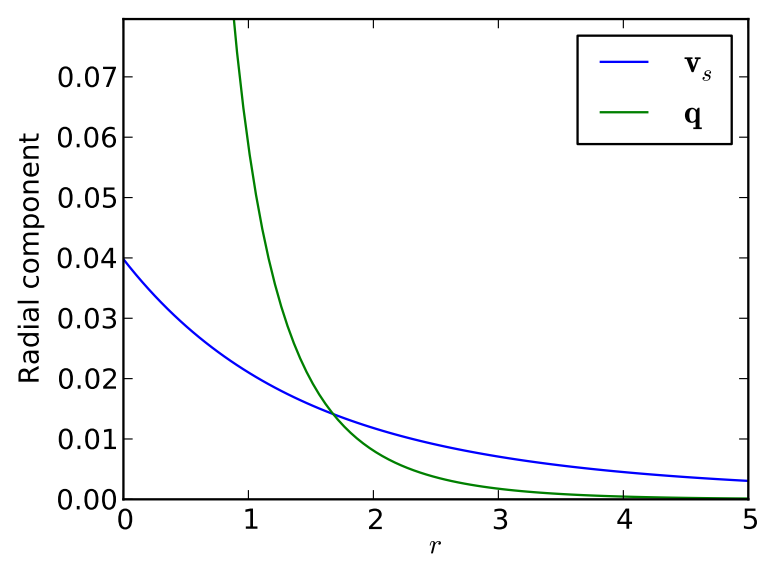

FigURE 1. Plot of the radial components of $\boldsymbol{v}_{s}$ and $\boldsymbol{q}$ for a point injection of melt of strength $Q=1$, as given by (4.15) and (4.16).

\section{A rigid impermeable sphere in a linear flow}

In this section we consider the case of a rigid impermeable sphere subjected to a far-field linear flow. The linear flow imposes far-field boundary conditions

$$
\boldsymbol{v}_{s} \rightarrow \boldsymbol{v}^{\infty}(\boldsymbol{x}), \quad P \rightarrow 0, \quad \text { as }|\boldsymbol{x}| \rightarrow \infty
$$

where $\boldsymbol{v}^{\infty}(\boldsymbol{x})$ is a general linear flow which can be decomposed into a translation, a rotation, and a strain,

$$
\boldsymbol{v}^{\infty}(\boldsymbol{x})=\boldsymbol{V}^{\infty}+\boldsymbol{\Omega}^{\infty} \times \boldsymbol{x}+E^{\infty} \cdot \boldsymbol{x} .
$$

$\boldsymbol{V}^{\infty}$ is the far-field translation vector, $\boldsymbol{\Omega}^{\infty}$ the far-field rotation vector, and $E^{\infty}$ is the symmetric second rank rate of strain tensor, which must be trace-free so that $\boldsymbol{v}^{\infty}(\boldsymbol{x})$ satisfies the compaction equations in an unbounded domain with $P=0$.

The sphere can undergo a rigid body rotation, but is taken to be impermeable. The boundary conditions on the sphere are thus

$$
\left.\boldsymbol{v}_{s}\right|_{r=a}=\boldsymbol{V}^{p}+\boldsymbol{\Omega}^{p} \times \boldsymbol{x},\left.\quad \boldsymbol{q} \cdot \boldsymbol{n}\right|_{r=a}=0,
$$

where $\boldsymbol{V}^{p}$ is the velocity of the sphere, and $\boldsymbol{\Omega}^{p}$ is its angular velocity. In order to solve the compaction equations subject to these boundary conditions, it is useful to recast the problem in terms of the perturbation to the background flow

$$
\tilde{\boldsymbol{v}}_{s}=\boldsymbol{v}_{s}-\boldsymbol{v}^{\infty}(\boldsymbol{x}), \quad \tilde{P}=P-0,
$$

which satisfies boundary conditions that decay at infinity,

$$
\tilde{\boldsymbol{v}}_{s} \rightarrow \mathbf{0}, \quad \tilde{P} \rightarrow 0, \quad \text { as }|\boldsymbol{x}| \rightarrow \infty,
$$

and on the sphere are

$$
\left.\tilde{\boldsymbol{v}}_{s}\right|_{r=a}=-\left(\boldsymbol{V}^{\infty}-\boldsymbol{V}^{p}\right)-\left(\boldsymbol{\Omega}^{\infty}-\boldsymbol{\Omega}^{p}\right) \times \boldsymbol{x}-E^{\infty} \cdot \boldsymbol{x},\left.\quad \tilde{\boldsymbol{q}} \cdot \boldsymbol{n}\right|_{r=a}=0 .
$$

Due to the linearity of the compaction equations, the above problem can be solved by a linear superposition of the solutions for the translating flow, the rotating flow, and the straining flow. In the next three subsections we consider each of these flows in turn. 


\subsection{Translating sphere}

For a rigid impermeable sphere translating at a velocity $-\boldsymbol{V}$ the boundary conditions on the sphere are

$$
\left.\boldsymbol{v}_{s=a}\right|_{r=-\boldsymbol{V}},\left.\quad \boldsymbol{q} \cdot \boldsymbol{n}\right|_{r=a}=0 .
$$

To find solutions satisfying these boundary conditions which decay at infinity we can use the Papkovich-Neuber potentials

$$
\begin{gathered}
\boldsymbol{\varphi}=\frac{C \boldsymbol{V}}{r}, \\
\chi=D \boldsymbol{V} \cdot \boldsymbol{\nabla}(1 / r)=-\frac{D \boldsymbol{V} \cdot \boldsymbol{x}}{r^{3}}, \\
\mathcal{C}=E \boldsymbol{V} \cdot \boldsymbol{\nabla}\left(k_{0}(r)\right)=-\frac{E k_{1}(r) \boldsymbol{V} \cdot \boldsymbol{x}}{r},
\end{gathered}
$$

which are educated guesses inspired by the following facts: The potentials must be linear in $\boldsymbol{V} ; \boldsymbol{\varphi}$ and $\chi$ must be harmonic and $\mathcal{C}$ must satisfy the modified Helmholtz equation; and the potentials must have the right behaviour under reflections (i.e. the potentials must be true tensors, not pseudo-tensors). $C, D$, and $E$ are unknown constants that will be determined by the boundary conditions. A potential of the form $\boldsymbol{V} \cdot \boldsymbol{\nabla} \boldsymbol{\nabla}(1 / r)$ could also have been used for $\varphi$, but it yields the same as the $\chi$ term (Papkovich-Neuber potentials are not unique, and any part of $\varphi$ that can be written as $\varphi=\nabla \vartheta$ with $\nabla^{2} \vartheta=0$ can also be written using a scalar potential $\chi)$.

Substituting (5.8-5.10) into (3.10) and (3.11) yields

$$
\begin{gathered}
\boldsymbol{v}_{s}=\left(-\frac{C}{r}-\frac{D}{r^{3}}-\frac{E k_{1}(r)}{r}\right) \boldsymbol{V}+\left(-\frac{C}{r^{3}}+\frac{3 D}{r^{5}}+\frac{E k_{2}(r)}{r^{2}}\right)(\boldsymbol{V} \cdot \boldsymbol{x}) \boldsymbol{x}, \\
P=\left(-\frac{2 \mathcal{B} C}{r^{3}}-\frac{E k_{1}(r)}{r}\right) \boldsymbol{V} \cdot \boldsymbol{x} .
\end{gathered}
$$

Other quantities of interest are given by

$$
\begin{gathered}
\boldsymbol{\omega}_{s}=\frac{2 C}{r^{3}} \boldsymbol{x} \times \boldsymbol{V} \\
\boldsymbol{q}=\left(\frac{2 \mathcal{B C}}{r^{3}}+\frac{E k_{1}(r)}{r}\right) \boldsymbol{V}+\left(-\frac{6 \mathcal{B} C}{r^{5}}-\frac{E k_{2}(r)}{r^{2}}\right)(\boldsymbol{V} \cdot \boldsymbol{x}) \boldsymbol{x}, \\
e=\left(-\frac{C}{r^{3}}+\frac{3 D}{r^{5}}+\frac{E k_{2}(r)}{r^{2}}\right)(\boldsymbol{V} \cdot \boldsymbol{x}) \boldsymbol{I}+\left(\frac{3 D}{r^{5}}+\frac{E k_{2}(r)}{r^{2}}\right)(\boldsymbol{V} \boldsymbol{x}+\boldsymbol{x} \boldsymbol{V}) \\
+\left(\frac{3 C}{r^{5}}-\frac{15 D}{r^{7}}-\frac{E k_{3}(r)}{r^{3}}\right)(\boldsymbol{V} \cdot \boldsymbol{x}) \boldsymbol{x} \boldsymbol{x}, \\
\bar{\sigma}=2 \mathcal{B}\left(\left(\frac{C}{r^{3}}+\frac{E k_{1}(r)}{r}\right)(\boldsymbol{V} \cdot \boldsymbol{x}) \boldsymbol{I}+e\right) .
\end{gathered}
$$

The boundary conditions (5.7) imply that

$$
\begin{gathered}
-\frac{C}{a}-\frac{D}{a^{3}}-\frac{E k_{1}(a)}{a}=-1, \\
-\frac{C}{a^{3}}+\frac{3 D}{a^{5}}+\frac{E k_{2}(a)}{a^{2}}=0, \\
-\frac{4 \mathcal{B} C}{a^{3}}+E k_{1}^{\prime}(a)=0,
\end{gathered}
$$




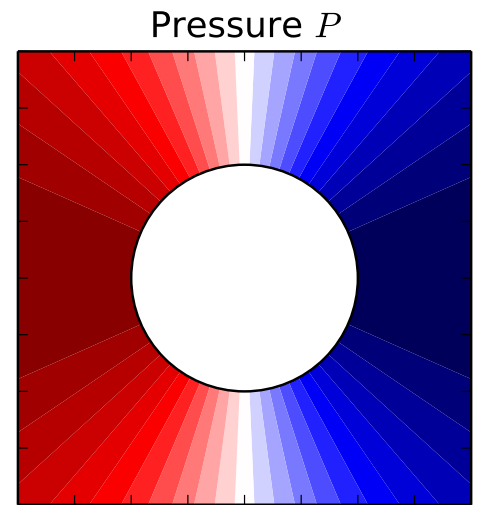

Compaction rate $\mathcal{C}$

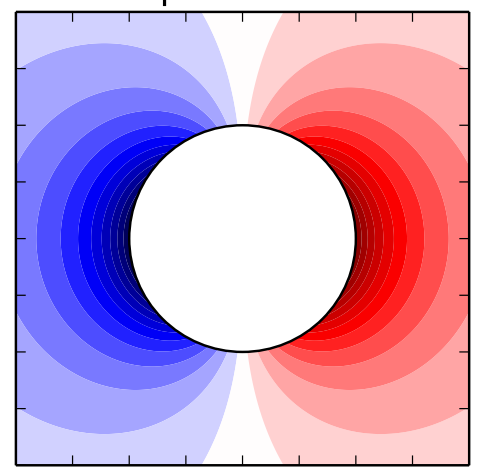

\section{Solid velocity $\mathbf{v}_{s}$}

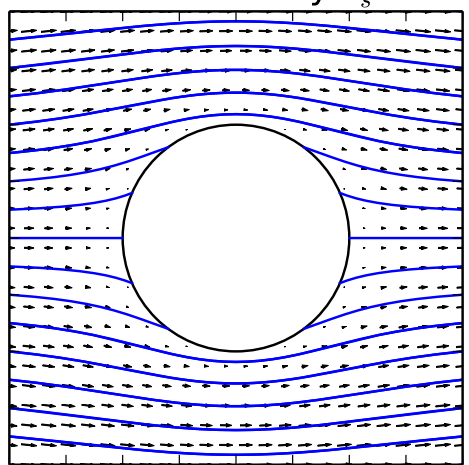

Darcy flux q

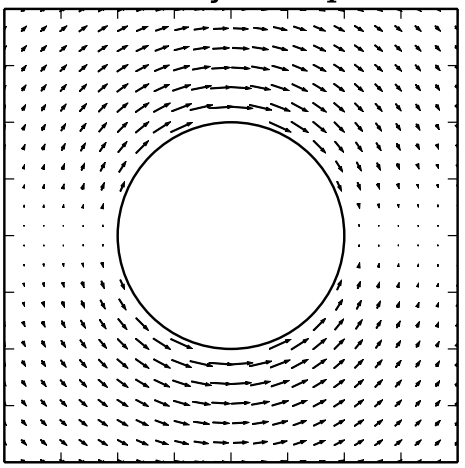

Figure 2. Pressure $P$, solid velocity $\boldsymbol{v}_{s}$, compaction rate $\mathcal{C}$, and Darcy flux $\boldsymbol{q}$ for uniform flow past a sphere. The sphere has radius $a=0.001$ in dimensionless units (i.e. the compaction length is large compared to the radius of the sphere). The bulk viscosity to shear viscosity ratio $R=1.0$. These plots are similar to those in figure 1 of McKenzie \& Holness (2000). Contours in the left hand plot are equally spaced with red indicating positive, blue indicating negative, and white zero. Matrix particle paths are shown as blue lines in the solid velocity plot. Arrows in the right hand plots show the direction and magnitude of the given velocity.

which can be solved to give

$$
\begin{gathered}
C=-\frac{3 a^{3} k_{1}^{\prime}(a)}{4\left(\mathcal{B} k_{0}(a)-a^{2} k_{1}^{\prime}(a)\right)}, \\
D=\frac{a^{3}\left(4 \mathcal{B} k_{2}(a)-a^{2} k_{1}^{\prime}(a)\right.}{4\left(\mathcal{B} k_{0}(a)-a^{2} k_{1}^{\prime}(a)\right)}, \\
E=-\frac{3 \mathcal{B}}{\mathcal{B} k_{0}(a)-a^{2} k_{1}^{\prime}(a)} .
\end{gathered}
$$

\subsubsection{Uniform flow past a sphere}

The above can be used to determine the solution for uniform flow past a sphere by adding on the far-field velocity $\boldsymbol{V}$. Flow patterns for uniform flow past a sphere are plotted in figures 2 and 3 for two different values of the ratio of sphere radius to compaction length. In both cases, on the side of the sphere facing the stream the matrix compacts and melt flows from this side of the sphere to the other. When the compaction length is large compared to the size of the sphere (figure 2), the compaction occurs over a region 


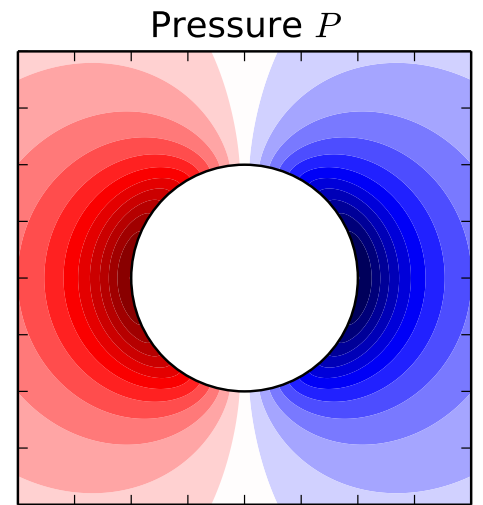

Compaction rate $\mathcal{C}$

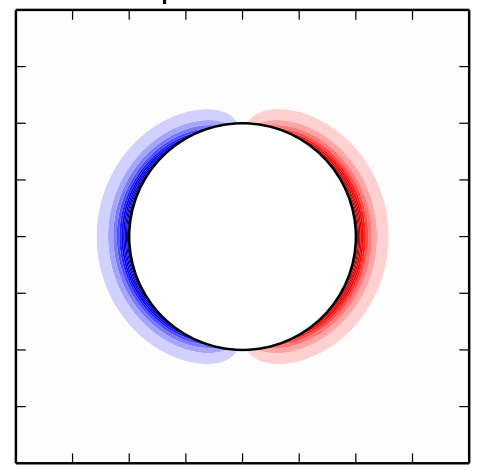

Solid velocity $\mathbf{v}_{s}$

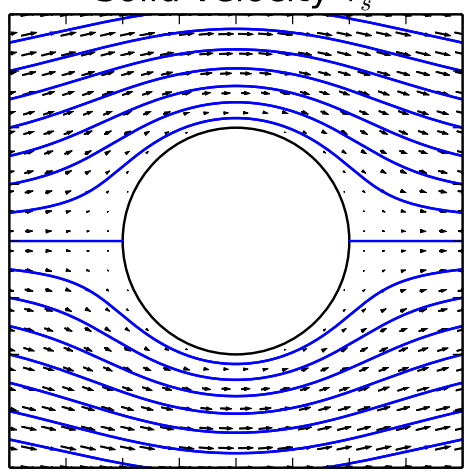

Darcy flux q

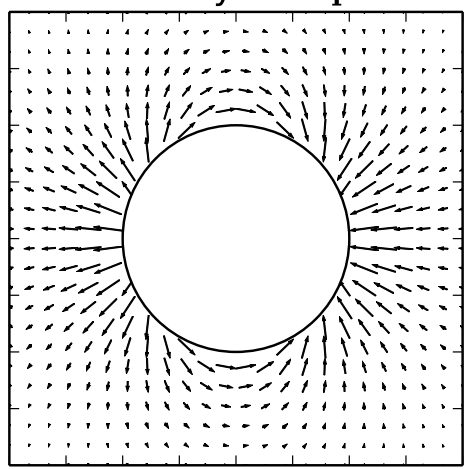

FiguRE 3. Uniform flow past a sphere as in figure 2, except now the sphere has radius $a=10.0$ in dimensionless units (i.e. the compaction length is small compared to the radius of the sphere). Compaction is confined to a small boundary layer around the sphere.

comparable in size to the radius of the sphere. When the compaction length is small compared to the size of the sphere (figure 3) compaction is confined to small boundary layers of a thickness on the order of the compaction length.

\subsubsection{Force on the sphere}

It is possible to calculate the force the flow exerts on the sphere. We first need the traction on the sphere, which is given from (5.16) as

$$
\left.\bar{\sigma} \cdot \boldsymbol{n}\right|_{r=a}=\frac{2 \mathcal{B} C}{a^{2}} \boldsymbol{V}
$$

The force on the sphere is then given by

$$
\boldsymbol{F} \equiv \int_{r=a} \bar{\sigma} \cdot \boldsymbol{n} \mathrm{d} S=8 \pi \mathcal{B} C \boldsymbol{V}=6 \pi \mathcal{B} a f(a) \boldsymbol{V}
$$

where

$$
f(a)=\frac{4 C}{3 a}=\frac{-a^{2} k_{1}^{\prime}(a)}{\mathcal{B} k_{0}(a)-a^{2} k_{1}^{\prime}(a)}=\frac{2+2 a+a^{2}}{2+2 a+a^{2}+\mathcal{B}} .
$$

(5.25) represents the amount by which the drag on the sphere is reduced by being in a deformable porous medium rather than an ordinary viscous fluid. $f(a)$ is plotted in figure 4. There are two important limits; when $a \gg 1$ (small compaction length), $f(a) \sim 1$, 


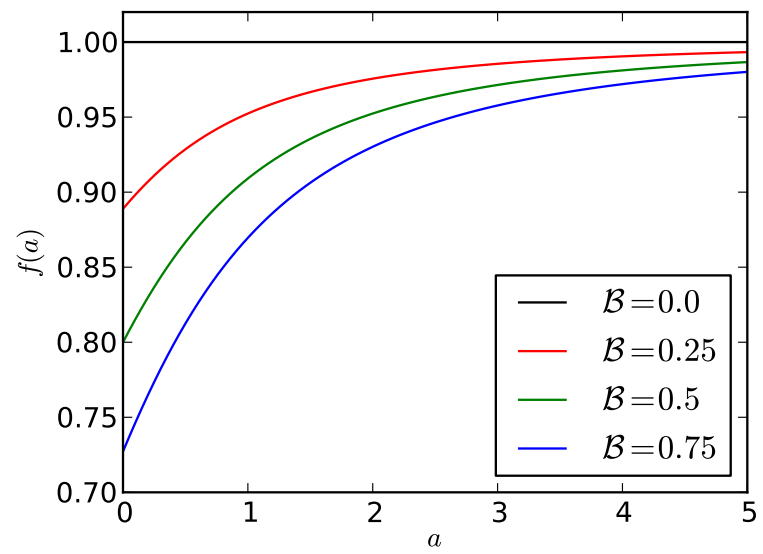

Figure 4. Plot of the drag reduction factor $f(a)$ as given in (5.25).

so (5.24) becomes

$$
\boldsymbol{F} \sim 6 \pi \mathcal{B} a \boldsymbol{V}
$$

which when converted back to dimensional form would be the usual Stokes drag law; and when $a \ll 1, f(a) \sim 2 /(2+\mathcal{B})$, so

$$
\boldsymbol{F} \sim \frac{12}{2+\mathcal{B}} \pi \mathcal{B} a \boldsymbol{V}
$$

which is the large compaction length limit and has a reduced drag. The maximum possible drag reduction from the usual Stokes law is $3 / 11 \approx 0.27$.

\subsubsection{Point force solution}

Taking the $a \rightarrow 0$ limit of the translating sphere solution for a fixed force gives the point force solution,

$$
\begin{gathered}
\boldsymbol{v}_{s}=\frac{1}{8 \pi \mathcal{B}}\left[\left(\frac{1}{r}+\frac{2 \mathcal{B}}{r^{3}}-\frac{2 \mathcal{B} k_{1}(r)}{r}\right) \boldsymbol{F}+\left(\frac{1}{r^{3}}-\frac{6 \mathcal{B}}{r^{5}}+\frac{2 \mathcal{B} k_{2}(r)}{r^{2}}\right)(\boldsymbol{F} \cdot \boldsymbol{x}) \boldsymbol{x}\right] \\
P=\frac{1}{4 \pi}\left(\frac{1}{r^{3}}-\frac{k_{1}(r)}{r}\right) \boldsymbol{F} \cdot \boldsymbol{x}, \\
\bar{\sigma}=-\frac{1}{2 \pi}\left[\left(\frac{3}{r^{5}}-\frac{k_{2}(r)}{r^{2}}-\frac{k_{1}(r)}{r}\right) \mathcal{B}(\boldsymbol{F} \cdot \boldsymbol{x}) I+\left(\frac{3}{r^{5}}-\frac{k_{2}(r)}{r^{2}}\right) \mathcal{B}(\boldsymbol{F} \boldsymbol{x}+\boldsymbol{x} \boldsymbol{F})\right. \\
\left.+\left(\frac{3}{2 r^{5}}-\frac{15 \mathcal{B}}{r^{7}}+\frac{\mathcal{B} k_{3}(r)}{r^{3}}\right)(\boldsymbol{F} \cdot \boldsymbol{x}) \boldsymbol{x} \boldsymbol{x}\right] .
\end{gathered}
$$

This solution formally satisfies

$$
-\nabla^{2} P+\nabla \cdot \boldsymbol{v}_{s}=0, \quad \boldsymbol{\nabla} \cdot \bar{\sigma}=-\boldsymbol{F} \delta^{(3)}(\boldsymbol{x}) .
$$

For $r \ll 1$ the Kelvinlet solution of the Navier-Cauchy equations is recovered (Phan- 
Thien \& Kim 1994),

$$
\begin{gathered}
\boldsymbol{v}_{s} \sim \frac{1}{8 \pi \mathcal{B}}\left(\frac{1+\mathcal{B}}{r} \boldsymbol{F}+\frac{1-\mathcal{B}}{r^{3}}(\boldsymbol{F} \cdot \boldsymbol{x}) \boldsymbol{x}\right), \\
P \sim \frac{\boldsymbol{F} \cdot \boldsymbol{x}}{8 \pi r}, \\
\bar{\sigma} \sim \frac{1}{4 \pi r^{3}}\left[\mathcal{B}((\boldsymbol{F} \cdot \boldsymbol{x}) \boldsymbol{I}-\boldsymbol{F} \boldsymbol{x}-\boldsymbol{x} \boldsymbol{F})-3(1-\mathcal{B})(\boldsymbol{F} \cdot \boldsymbol{x}) \frac{\boldsymbol{x} \boldsymbol{x}}{r^{2}}\right],
\end{gathered}
$$

and for $r \gg 1$ the Stokeslet solution of the Stokes equations is recovered (Guazzelli \& Morris 2012; Kim \& Karrila 2005),

$$
\begin{gathered}
\boldsymbol{v}_{s} \sim \frac{1}{8 \pi \mathcal{B}}\left(\frac{\boldsymbol{F}}{r}+\frac{(\boldsymbol{F} \cdot \boldsymbol{x}) \boldsymbol{x}}{r^{3}}\right), \\
P \sim \frac{\boldsymbol{F} \cdot \boldsymbol{x}}{4 \pi r^{3}} \\
\bar{\sigma} \sim-\frac{3(\boldsymbol{F} \cdot \boldsymbol{x}) \boldsymbol{x} \boldsymbol{x}}{4 \pi r^{5}} .
\end{gathered}
$$

The Stokeslet solution is considered to be one of the fundamental solutions of the Stokes equations, and is used as the basis for Green's function techniques for solving the Stokes flow equations, such as the boundary element method. The solution (5.28-5.30) is the generalisation of the Stokeslet to the compaction equations.

\subsection{Rotating sphere}

For a sphere rotating at an angular velocity $-\boldsymbol{\Omega}$ the boundary conditions on the sphere are

$$
\left.\boldsymbol{v}_{s}\right|_{r=a}=-\boldsymbol{\Omega} \times \boldsymbol{x},\left.\quad \boldsymbol{q} \cdot \boldsymbol{n}\right|_{r=a}=0 .
$$

The solution with these boundary conditions does not involve compaction and is unchanged from the usual Stokes flow problem. The only Papkovich-Neuber potential needed is

$$
\varphi \propto \Omega \times \nabla(1 / r)=-\frac{\boldsymbol{\Omega} \times \boldsymbol{x}}{r^{3}}
$$

and the solution is given by

$$
\begin{gathered}
\boldsymbol{v}_{s}=-\frac{a^{3}}{r^{3}} \boldsymbol{\Omega} \times \boldsymbol{x}, \\
P=0, \\
\boldsymbol{\omega}_{s}=a^{3}\left(\frac{\boldsymbol{\Omega}}{r^{3}}-\frac{3(\boldsymbol{\Omega} \cdot \boldsymbol{x}) \boldsymbol{x}}{r^{5}}\right), \\
\boldsymbol{q}=\mathbf{0}, \\
e=\frac{3 a^{3}}{2 r^{5}}((\boldsymbol{\Omega} \times \boldsymbol{x}) \boldsymbol{x}+\boldsymbol{x}(\boldsymbol{\Omega} \times \boldsymbol{x})), \\
\bar{\sigma}=2 \mathcal{B} e .
\end{gathered}
$$

In particular, the traction on the sphere is given by

$$
\left.\bar{\sigma} \cdot \boldsymbol{n}\right|_{r=a}=\frac{3 \mathcal{B}}{a} \boldsymbol{\Omega} \times \boldsymbol{x}
$$


and hence the torque on the sphere can be calculated as

$$
\boldsymbol{G} \equiv \int_{r=a} \boldsymbol{x} \times \bar{\sigma} \cdot \boldsymbol{n} \mathrm{d} S=8 \pi \mathcal{B} a^{3} \boldsymbol{\Omega}
$$

\subsection{Sphere under pure strain}

For the pure strain part of the flow (5.6) the boundary conditions on the sphere are

$$
\left.\boldsymbol{v}_{s}\right|_{r=a}=-E \cdot \boldsymbol{x},\left.\quad \boldsymbol{q} \cdot \boldsymbol{n}\right|_{r=a}=0 .
$$

Linearity suggests Papkovich-Neuber potentials of the form

$$
\begin{gathered}
\varphi=F E \cdot \nabla(1 / r)=-\frac{F E \cdot \boldsymbol{x}}{r^{3}}, \\
\chi=G E: \nabla \nabla(1 / r)=\frac{3 G \boldsymbol{x} \cdot E \cdot \boldsymbol{x}}{r^{5}}, \\
\mathcal{C}=H E: \nabla \nabla\left(k_{0}(r)\right)=\frac{H k_{2}(r) \boldsymbol{x} \cdot E \cdot \boldsymbol{x}}{r^{2}},
\end{gathered}
$$

for unknown constants $F, G$ and $H$. A potential of the form $E: \nabla \nabla \nabla(1 / r)$ could also have been used for $\varphi$ but this yields the same result as the $\chi$ does (again, PapkovichNeuber potentials are not unique).

Substituting into (3.10) and (3.11) yields

$$
\begin{gathered}
\boldsymbol{v}_{s}=\left(\frac{6 G}{r^{5}}+\frac{2 H k_{2}(r)}{r^{2}}\right) E \cdot \boldsymbol{x}+\left(\frac{3 F}{r^{5}}-\frac{15 G}{r^{7}}-\frac{H k_{3}(r)}{r^{3}}\right)(\boldsymbol{x} \cdot E \cdot \boldsymbol{x}) \boldsymbol{x} \\
P=\left(\frac{6 \mathcal{B F}}{r^{5}}+\frac{H k_{2}(r)}{r^{2}}\right) \boldsymbol{x} \cdot E \cdot \boldsymbol{x} .
\end{gathered}
$$

Other quantities of interest are given by

$$
\begin{aligned}
\boldsymbol{\omega}_{s}= & -\frac{6 F}{r^{5}} \boldsymbol{x} \times E \cdot \boldsymbol{x} \\
\boldsymbol{q}= & \left(-\frac{12 \mathcal{B} F}{r^{5}}-\frac{2 H k_{2}(r)}{r^{2}}\right) E \cdot \boldsymbol{x}+\left(\frac{30 \mathcal{B} F}{r^{7}}+\frac{H k_{3}(r)}{r^{3}}\right)(\boldsymbol{x} \cdot E \cdot \boldsymbol{x}) \boldsymbol{x} \\
e= & \left(\frac{6 G}{r^{5}}+\frac{2 k_{2}(r) H}{r^{2}}\right) E \\
& +\left(\frac{3 F}{r^{5}}-\frac{30 G}{r^{7}}-\frac{2 H k_{3}(r)}{r^{3}}\right)((E \cdot \boldsymbol{x}) \boldsymbol{x}+\boldsymbol{x}(E \cdot \boldsymbol{x})) \\
& +\left(\frac{3 F}{r^{5}}-\frac{15 G}{r^{7}}-\frac{H k_{3}(r)}{r^{3}}\right)(\boldsymbol{x} \cdot E \cdot \boldsymbol{x}) \boldsymbol{I} \\
& +\left(-\frac{15 F}{r^{7}}+\frac{105 G}{r^{9}}+\frac{H k_{4}(r)}{r^{4}}\right)(\boldsymbol{x} \cdot E \cdot \boldsymbol{x}) \boldsymbol{x} \boldsymbol{x} \\
\bar{\sigma}= & 2 \mathcal{B}\left(-\left(\frac{3 F}{r^{5}}+\frac{H k_{2}(r)}{r^{2}}\right)(\boldsymbol{x} \cdot E \cdot \boldsymbol{x}) \boldsymbol{I}+e\right)
\end{aligned}
$$


The boundary conditions (5.48) imply that

$$
\begin{gathered}
\frac{6 G}{a^{5}}+\frac{2 H k_{2}(a)}{a^{2}}=-1, \\
\frac{3 F}{a^{5}}-\frac{15 G}{a^{7}}-\frac{H k_{3}(a)}{a^{3}}=0, \\
\frac{18 \mathcal{B} F}{a^{5}}-\frac{H k_{2}^{\prime}(a)}{a}=0,
\end{gathered}
$$

and thus

$$
\begin{gathered}
F=\frac{5 a^{5} k_{2}^{\prime}(a)}{6\left(6 \mathcal{B} k_{1}(a)-a^{2} k_{2}^{\prime}(a)\right)}, \\
G=-\frac{a^{5}}{6}-\frac{5 a^{4} \mathcal{B} k_{2}(a)}{6 \mathcal{B} k_{1}(a)-a^{2} k_{2}^{\prime}(a)}, \\
H=\frac{15 a \mathcal{B}}{6 \mathcal{B} k_{1}(a)-a^{2} k_{2}^{\prime}(a)} .
\end{gathered}
$$

\subsubsection{A sphere in simple shear}

The solution above can be used to calculate the compacting flow round a sphere placed in simple shear. Simple shear flow has $\boldsymbol{v}^{\infty}(\boldsymbol{x})=(\gamma y, 0,0)$ and can be decomposed using the notation of $(5.2)$ as $\boldsymbol{V}^{\infty}=\mathbf{0}, \boldsymbol{\Omega}^{\infty}=(0,0,-\gamma / 2)$, and

$$
E^{\infty}=\left(\begin{array}{ccc}
0 & \gamma / 2 & 0 \\
\gamma / 2 & 0 & 0 \\
0 & 0 & 0
\end{array}\right)
$$

If the sphere is allowed to move freely, there must be no net force or torque on the sphere. This means that the sphere does not translate $\boldsymbol{V}^{p}=\mathbf{0}$, and rotates at the same rate as the far field flow $\boldsymbol{\Omega}^{p}=\boldsymbol{\Omega}^{\infty}$. The problem for the perturbation field is then simply that of pure strain as given in the previous section.

Figures 5 and 6 show plots of the solution to simple shear flow past a sphere. Again, for large compaction lengths the compaction occurs on distances of the order of the radius of the sphere, whereas for small compaction lengths the compaction is confined to small boundary layers. The compaction rate shows a distinctive quadrupole pattern, as is expected from the symmetry of the problem. The region of closed matrix pathlines indicates rotation of material near the sphere.

\subsubsection{Stresslet on the sphere}

In the same way that the force and torque have been calculated for the translating and rotating spheres, an appropriate moment of the traction called the stresslet can be calculated to describe the action of straining. In pure straining flow the traction around the sphere is given from (5.57) as

$$
\left.\bar{\sigma} \cdot \boldsymbol{n}\right|_{r=a}=-\frac{6 \mathcal{B} F}{a^{4}} E \cdot \boldsymbol{x},
$$

where the far field flow $E \cdot \boldsymbol{x}$ has been added back on to $\boldsymbol{v}_{s}$ so that $\left.\boldsymbol{v}_{s}\right|_{r=a}=\mathbf{0}$ and $\boldsymbol{v}_{s} \rightarrow E \cdot \boldsymbol{x}$ as $|\boldsymbol{x}| \rightarrow \infty$.

The stresslet on the sphere is given by the symmetric part of the first moment of traction,

$$
S \equiv \frac{1}{2} \int_{r=a} \boldsymbol{x}(\bar{\sigma} \cdot \boldsymbol{n})+(\bar{\sigma} \cdot \boldsymbol{n}) \boldsymbol{x} \mathrm{d} S=-8 \pi \mathcal{B} F E=\frac{20}{3} \mathcal{B} \pi a^{3} g(a) E
$$




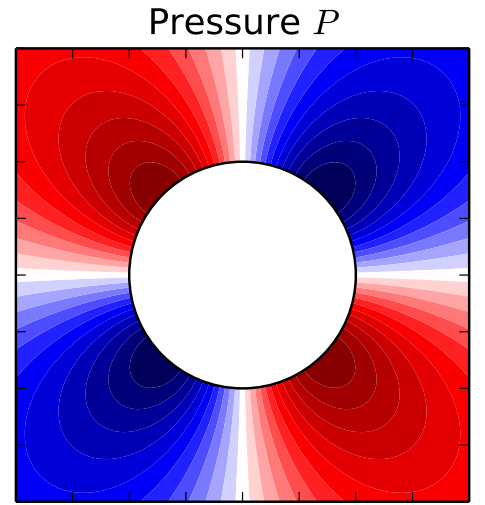

Compaction rate $\mathcal{C}$

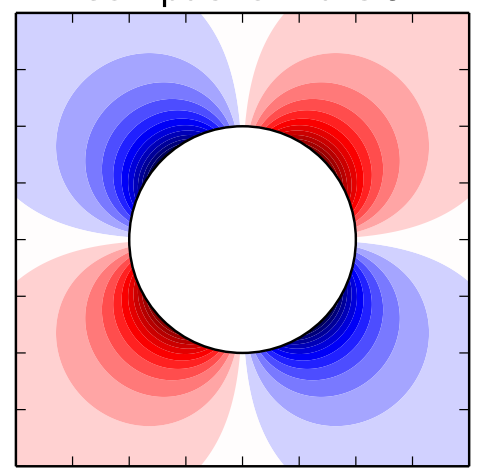

\section{Solid velocity $\mathbf{v}_{s}$}

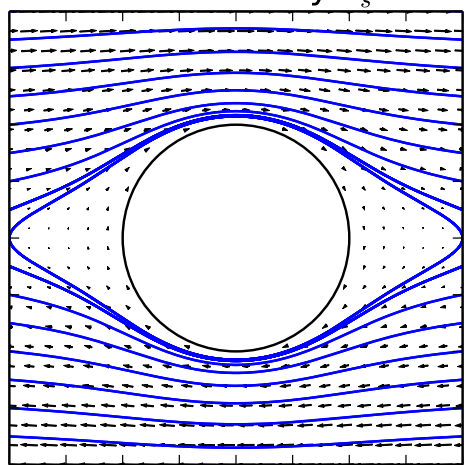

Darcy flux q

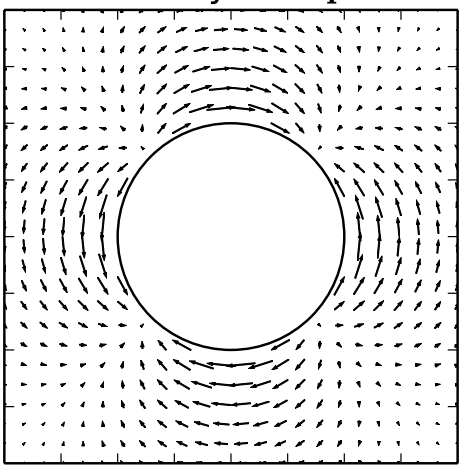

FiguRE 5. Freely rotating sphere in a simple shear flow, with fields as in figures 2 and 3 . The sphere has radius $a=0.001$ and $R=1.0$. The far field simple shear takes the form $\boldsymbol{v}_{s} \sim(\gamma y, 0,0)$. Shown are plots of the $x-y$ plane.

where

$$
g(a)=-\frac{6 F}{5 a^{3}}=\frac{-a^{2} k_{2}^{\prime}(a)}{6 \mathcal{B} k_{1}(a)-a^{2} k_{2}^{\prime}(a)}=\frac{9+9 a+4 a^{2}+a^{3}}{9+9 a+4 a^{2}+a^{3}+6 \mathcal{B}(1+a)} .
$$

$g(a)$ is similar to $f(a)$ defined in (5.25) in that it defines the reduction in the stresslet from the usual Stokes flow expression. Again, there are two important limits; when $a \gg 1$, $g(a) \sim 1$ and

$$
S \sim \frac{20}{3} \mathcal{B} \pi a^{3} E
$$

which when converted back into dimensional units is the usual result for Stokes flow; and when $a \ll 1, g(a) \sim 3 /(3+2 \mathcal{B})$ and

$$
S \sim \frac{20}{2 \mathcal{B}+3} \mathcal{B} \pi a^{3} E
$$

which is the large compaction length limit and is smaller. The maximum possible reduction in the stresslet from the usual Stokes flow expression is $1 / 3$.

\section{Faxén relations}

The expressions found in the previous sections for the force, torque, and stresslet on a rigid sphere in a linear flow can be extended to give the force, torque, and stresslet 


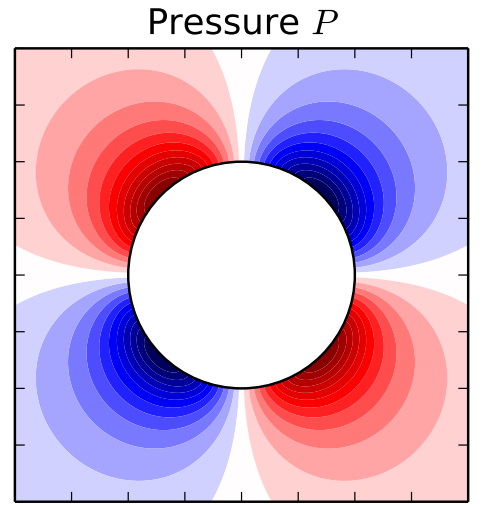

Compaction rate $\mathcal{C}$

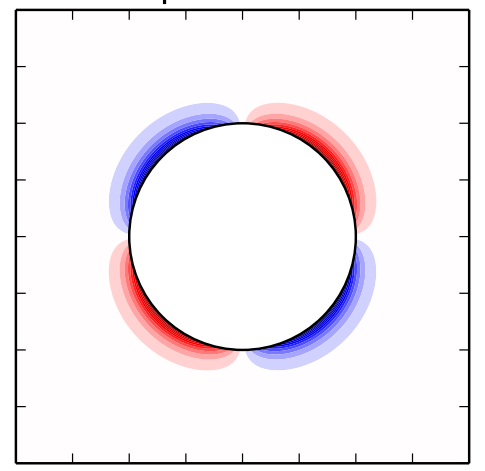

Solid velocity $\mathbf{v}_{s}$

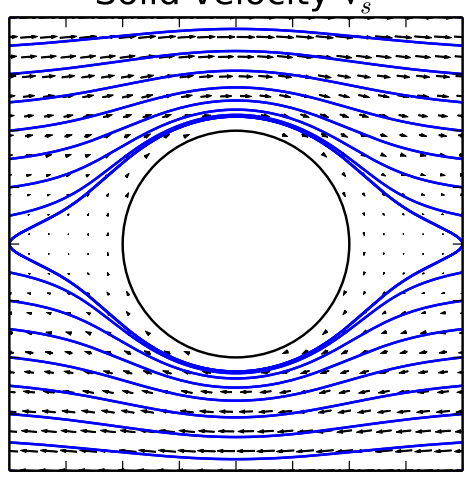

Darcy flux q

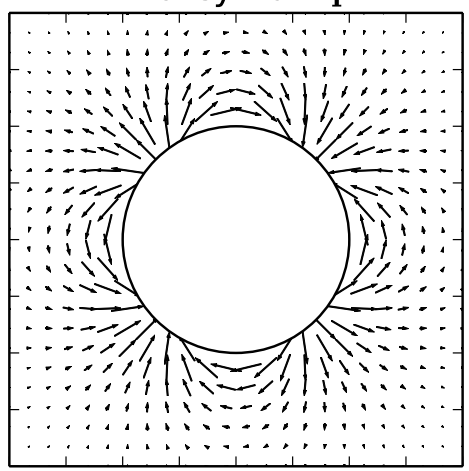

FiguRE 6. Freely rotating sphere in a simple shear flow as in figure 5, except now the sphere has radius $a=10.0$. As before, $R=1.0$.

on a sphere in an arbitrary flow (including quadratic flows such as Poiseuille flow). For Stokes flow, these expressions are called the Faxén relations. They can be derived by substituting a series of test flows into the reciprocal theorem (Kim \& Karrila 2005), and this approach can be generalised to the compaction equations.

\subsection{A reciprocal theorem}

If $\left\{\tilde{\boldsymbol{v}}_{s}, \tilde{P}, \tilde{\boldsymbol{q}}\right\}$ is one solution of the compaction equations and $\left\{\boldsymbol{v}_{s}^{\prime}, P^{\prime}, \boldsymbol{q}^{\prime}\right\}$ is another on the same domain but with potentially different boundary conditions, then they satisfy the following reciprocal theorem (Appendix A)

$$
\int_{S}-\tilde{P} \boldsymbol{q}^{\prime} \cdot \boldsymbol{n}+\boldsymbol{v}_{s}^{\prime} \cdot \tilde{\bar{\sigma}} \cdot \boldsymbol{n} \mathrm{d} S=\int_{S}-P^{\prime} \tilde{\boldsymbol{q}} \cdot \boldsymbol{n}+\tilde{\boldsymbol{v}}_{s} \cdot \bar{\sigma}^{\prime} \cdot \boldsymbol{n} \mathrm{d} S
$$

where $S$ is the surface bounding the domain of interest. To derive the Faxén relations we can use a weaker form of this reciprocal theorem. If we just consider those flows which vanish at infinity around a sphere that is rigid and impermeable, then $\left.\boldsymbol{q}^{\prime} \cdot \boldsymbol{n}\right|_{r=a}=$ $\left.\tilde{\boldsymbol{q}} \cdot \boldsymbol{n}\right|_{r=a}=0$, and the surface integral is only that over the sphere

$$
\int_{r=a} \boldsymbol{v}_{s}^{\prime} \cdot \tilde{\bar{\sigma}} \cdot \boldsymbol{n} \mathrm{d} S=\int_{r=a} \tilde{\boldsymbol{v}}_{s} \cdot \bar{\sigma}^{\prime} \cdot \boldsymbol{n} \mathrm{d} S
$$


Consider a sphere placed in an arbitrary compacting flow $\boldsymbol{v}^{\infty}(\boldsymbol{x})$, not necessarily linear. Suppose the sphere undergoes a rigid body motion, so that

$$
\left.\boldsymbol{v}_{s}\right|_{r=a}=\boldsymbol{V}_{p}+\boldsymbol{\Omega}_{p} \times \boldsymbol{x}, \quad \boldsymbol{v}_{s} \rightarrow \boldsymbol{v}^{\infty}(\boldsymbol{x}) \text { as } r \rightarrow \infty .
$$

The perturbation flow $\tilde{\boldsymbol{v}}_{s}=\boldsymbol{v}_{s}-\boldsymbol{v}^{\infty}$ satisfies

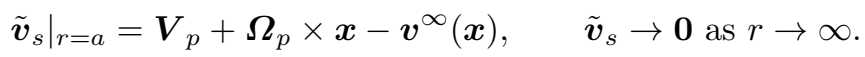

Substituting into the reciprocal theorem (6.2) yields

$$
\int_{r=a} \boldsymbol{v}_{s}^{\prime} \cdot \bar{\sigma} \cdot \boldsymbol{n} \mathrm{d} S=\int_{r=a} \boldsymbol{v}_{s}^{\prime} \cdot \bar{\sigma}^{\infty} \cdot \boldsymbol{n} \mathrm{d} S+\int_{r=a}\left(\boldsymbol{V}_{p}+\boldsymbol{\Omega}_{p} \times \boldsymbol{x}-\boldsymbol{v}^{\infty}(\boldsymbol{x})\right) \cdot \bar{\sigma}^{\prime} \cdot \boldsymbol{n} \mathrm{d} S .
$$

The Faxén relations for the force, torque, and stresslet are obtained by substituting a series of different test flows into the $\boldsymbol{v}_{s}^{\prime}$ of $(6.5)$.

\subsection{Force on a sphere in an arbitrary flow}

Consider as a test flow $\boldsymbol{v}_{s}^{\prime}$ the flow due to translation at a uniform velocity $\boldsymbol{V}^{\prime}$, as solved in subsection 5.1. This satisfies

$$
\left.\boldsymbol{v}_{s}^{\prime}\right|_{r=a}=-\boldsymbol{V}^{\prime},\left.\quad \bar{\sigma}^{\prime} \cdot \boldsymbol{n}\right|_{r=a}=\frac{3 f(a) \mathcal{B}}{2 a} \boldsymbol{V}^{\prime},
$$

where $f(a)$ is the drag reduction factor given in (5.25). Applying the reciprocal theorem (6.5), and noting that $\boldsymbol{V}^{\prime}$ is arbitrary, we obtain a Faxén law for the force on the sphere,

$$
\boldsymbol{F}=6 \pi \mathcal{B} a f(a)\left(\frac{1}{4 \pi a^{2}} \int_{r=a} \boldsymbol{v}^{\infty}(\boldsymbol{x}) \mathrm{d} S-\boldsymbol{V}_{p}\right) .
$$

\subsection{Torque on a sphere in an arbitrary flow}

Now consider as a test flow that due to a rotating sphere with a uniform angular velocity $\boldsymbol{\Omega}^{\prime}$, as solved in subsection 5.2. This satisfies

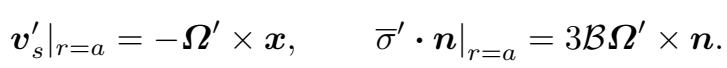

Applying the reciprocal theorem (6.5), and noting that $\boldsymbol{\Omega}^{\prime}$ is arbitrary, we obtain a Faxén law for the torque on the sphere,

$$
\boldsymbol{G}=4 \pi \mathcal{B} a^{3}\left(\frac{3}{4 \pi a^{3}} \int_{r=a} \boldsymbol{n} \times \boldsymbol{v}^{\infty}(\boldsymbol{x}) \mathrm{d} S-2 \boldsymbol{\Omega}_{p}\right) .
$$

\subsection{Stresslet on a sphere in an arbitrary flow}

To derive the Faxén laws for the stresslet it is helpful to consider the volumetric and deviatoric (trace-free) parts separately. We write the stresslet $S=\hat{S}+\tilde{S}$ where $\hat{S}$ is the volumetric part and $\tilde{S}$ is the deviatoric part. Note that

$$
\begin{gathered}
\hat{S}=\frac{1}{3} I \operatorname{tr}(S), \\
\tilde{S}=S-\frac{1}{3} I \operatorname{tr}(S) .
\end{gathered}
$$

To derive the Faxén law for the volumetric part of the stresslet, consider a test flow consisting of pure expansion/contraction as solved in subsection 4.1. This satisfies

$$
\left.\boldsymbol{v}_{s}^{\prime}\right|_{r=a}=\frac{Q^{\prime}}{4 \pi a^{3}} \boldsymbol{x},\left.\quad \bar{\sigma}^{\prime} \cdot \boldsymbol{n}\right|_{r=a}=-\frac{\mathcal{B} Q^{\prime} \boldsymbol{n}}{\pi a^{3}} .
$$


Applying the reciprocal theorem (6.5), and noting that $Q^{\prime}$ is arbitrary, we find that the volumetric part of the stresslet is

$$
\hat{S}=I \int_{r \leq a} \mathcal{C}^{\infty}(\boldsymbol{x})-P^{\infty}(\boldsymbol{x}) \mathrm{d} V,
$$

where we have exploited the identities

$$
\begin{gathered}
\int_{r=a} \boldsymbol{x} \bar{\sigma}^{\infty} \cdot \boldsymbol{n} \mathrm{d} S=\int_{r \leq a} \bar{\sigma}^{\infty} \mathrm{d} V \\
\bar{\sigma}^{\infty}=-P^{\infty} I+\left(1-\frac{4 \mathcal{B}}{3}\right) \mathcal{C}^{\infty} I+\mathcal{B}\left(\boldsymbol{\nabla} \boldsymbol{v}^{\infty}+\left(\nabla \boldsymbol{v}^{\infty}\right)^{T}-\frac{2}{3} \mathcal{C}^{\infty} \boldsymbol{I}\right) .
\end{gathered}
$$

To derive the Faxén law for the deviatoric part of the stresslet, consider a test flow which has a uniform strain on the surface on the sphere, as solved in subsection 5.3,

$$
\left.\boldsymbol{v}_{s}^{\prime}\right|_{r=a}=-E^{\prime} \cdot \boldsymbol{x},\left.\quad \bar{\sigma}^{\prime} \cdot \boldsymbol{n}\right|_{r=a}=2(-1+5 g(a)) \mathcal{B} E^{\prime} \cdot \boldsymbol{n},
$$

where $g(a)$ is given by (5.67). Applying the reciprocal theorem (6.5), and noting that $E^{\prime}$ is arbitrary, we find the deviatoric part of the stresslet is

$$
\tilde{S}=\frac{20}{3} \mathcal{B} \pi a^{3} g(a)\left(\frac{3}{4 \pi a^{3}} \int_{r=a} \frac{\boldsymbol{n v} \boldsymbol{v}^{\infty}(\boldsymbol{x})+\boldsymbol{v}^{\infty}(\boldsymbol{x}) \boldsymbol{n}}{2} \mathrm{~d} S-\frac{\boldsymbol{I}}{4 \pi a^{3}} \int_{r=a} \boldsymbol{n} \cdot \boldsymbol{v}^{\infty}(\boldsymbol{x}) \mathrm{d} S\right) .
$$

\subsection{Mean value integrals}

The Faxén relations given in (6.7), (6.9), (6.13), and (6.17) involve averages of the farfield velocity $\boldsymbol{v}^{\infty}(\boldsymbol{x})$ over the sphere. These averages can be simplified and written in terms of derivatives of the far-field velocity at the origin using mean value theorems for solutions of Laplace's equation, the biharmonic equation, and the modified Helmholtz equation (e.g. Poritsky (1938); Sabelfeld \& Shalimova (1997)).

The Faxén force law (6.7) can be simplified using the fact that $\boldsymbol{v}_{s}-\nabla \mathcal{C}$ satisfies the biharmonic equation and that $\nabla \mathcal{C}$ satisfies the modified Helmholtz equation. The mean value integral in (6.7) becomes

$$
\frac{1}{4 \pi a^{2}} \int_{r=a} \boldsymbol{v}^{\infty}(\boldsymbol{x}) \mathrm{d} S=\left(1+\frac{a^{2}}{6} \nabla^{2}\right) \boldsymbol{v}^{\infty}(\mathbf{0})+\left(i_{0}(a)-1-\frac{a^{2}}{6}\right) \nabla \mathcal{C}^{\infty}(\mathbf{0})
$$

where $i_{0}(a)$ is a modified spherical Bessel function of the first kind, $i_{0}(a)=\sinh (a) / a$.

The Faxén torque law (6.9) can be simplified using the fact that the solid vorticity is harmonic, which implies that

$$
\frac{3}{4 \pi a^{3}} \int_{r=a} \boldsymbol{n} \times \boldsymbol{v}^{\infty}(\boldsymbol{x}) \mathrm{d} S=\boldsymbol{\nabla} \times \boldsymbol{v}^{\infty}(\mathbf{0})=\boldsymbol{\omega}^{\infty}(\mathbf{0}),
$$

where $\boldsymbol{\omega}^{\infty}(\boldsymbol{x})$ is the solid vorticity of the far-field flow.

The Faxén stresslet laws (6.13) and (6.17) can be simplified in a similar way using

$$
\begin{aligned}
& \frac{3}{4 \pi a^{3}} \int_{r \leq a} \mathcal{C}^{\infty}(\boldsymbol{x})-P^{\infty}(\boldsymbol{x}) \mathrm{d} V=\mathcal{C}^{\infty}(\mathbf{0})-P^{\infty}(\mathbf{0}) \\
& \frac{1}{4 \pi a^{2}} \int_{r=a} \boldsymbol{n} \cdot \boldsymbol{v}^{\infty}(\boldsymbol{x}) \mathrm{d} S=i_{1}(a) \mathcal{C}^{\infty}(\mathbf{0}),
\end{aligned}
$$

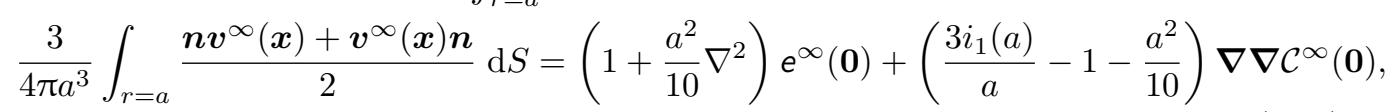


where

$$
i_{1}(a)=\frac{\mathrm{d} i_{0}(a)}{\mathrm{d} a}=\frac{a \cosh (a)-\sinh (a)}{a^{2}} .
$$

\section{Porosity evolution}

Thus far we have been concerned with the instantaneous solution of the compaction equations for a uniform medium, and have been able to produce a wide variety of analytical solutions. However, solving for the full porosity evolution over time requires a numerical solution, for two main reasons: First, even if we have an exact solution for $\boldsymbol{v}_{s}$, the porosity evolution equation (2.1) is difficult to integrate analytically. Moreover, because $k_{\phi}, \eta_{\phi}$, and $\zeta_{\phi}$ are all typically functions of porosity, the velocity field evolves with the porosity field making the problem highly non-linear. Nevertheless, we can get some insight into the porosity evolution by assuming the velocity field $\boldsymbol{v}_{s}$ remains constant over time. This is expected to be a good approximation when the variations in porosity are small, so that the functions $k_{\phi}, \eta_{\phi}$, and $\zeta_{\phi}$ remain approximately constant. The porosity evolution can then obtained from integration of (2.1) which can be recast in the form

$$
\frac{\mathrm{D}_{s}}{\mathrm{D} t} \log (1-\phi)=-\mathcal{C}
$$

where $\frac{\mathrm{D}_{s}}{\mathrm{D} t} \equiv \frac{\partial}{\partial t}+\boldsymbol{v}_{s} \cdot \boldsymbol{\nabla}$ represents a Lagrangian derivative along a matrix particle path. Along such a path, (7.1) represents a simple ODE for the porosity evolution and is straightforward to integrate numerically.

Figures 7 and 8 show two plots of the porosity evolution calculated by numerically integrating (7.1) for a translating sphere. Figure 7 is a large compaction length case, figure 8 is a small compaction length case. In both figures the ratio of bulk viscosity to shear viscosity $R=1000$, chosen to be large so that the porosity variations remain small. For short times the porosity field mirrors the compaction rate field with its distinctive dipole pattern (figures 2 and 3). This is to be expected, because for short times there will be little movement along a matrix particle path, and the porosity evolution will simply reflect the right-hand side of (7.1) given by the compaction rate. For later times the effect of advection is clearly visible, and an asymmetry develops between the regions of high porosity and low porosity. The low porosity compacting region facing the stream becomes wider and the high porosity de-compacting region on the opposite side becomes narrower.

Figures 9 and 10 show similar plots of the porosity evolution for a sphere in simple shear. Figure 9 is a large compaction length case, figure 10 is a small compaction length case. Again, for short times the porosity field mirrors the compaction rate field with its distinctive quadrupole pattern (figures 5 and 6 ). The rotation of the flow is clearly visible, and again the low porosity regions become wider as the high porosity regions become narrower. After the sphere has rotated by $\pi$ radians, the surface of the sphere is back at the initial porosity, as along each matrix pathline an equal amount of time has been spent compacting and de-compacting.

\section{Conclusions}

A series of analytical solutions have been found for compacting flow past a sphere. These solutions generalise the well-known results for Stokes flow, and are valid for an arbitrary compaction length. The solutions are limited in use in that they are only valid instantaneously for a uniform medium, and can only be used to provide an approximate 

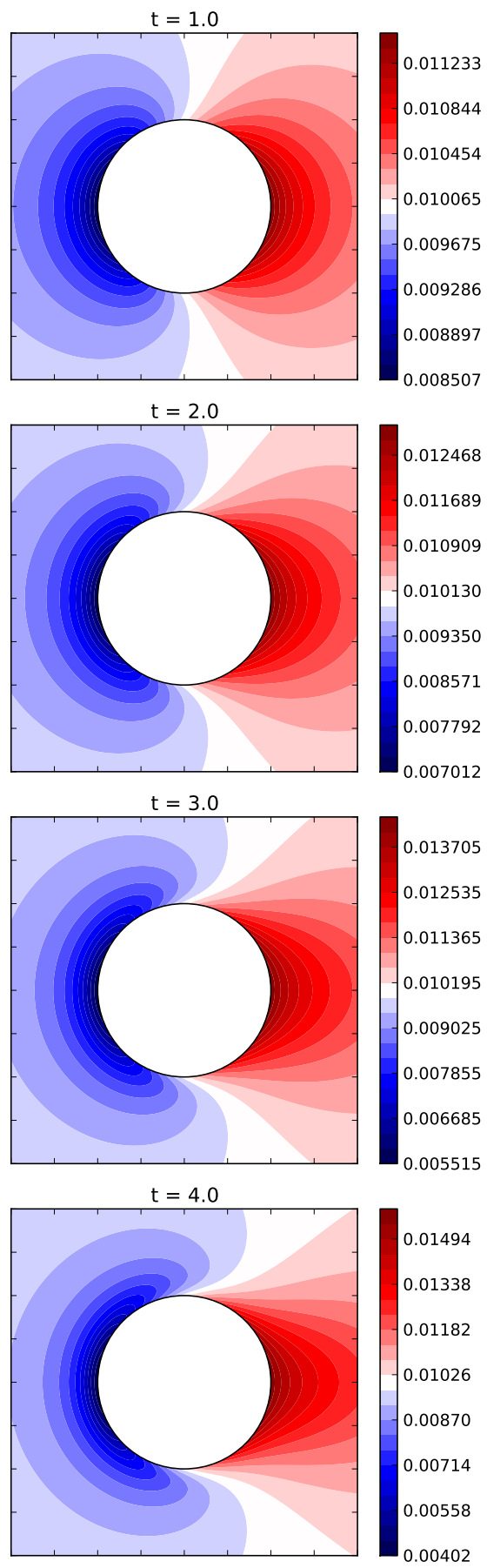

FiguRE 7. Plots showing porosity evolution around a translating sphere. The far field flow is the same as in figures 2 and 3 . The sphere has radius $a=0.001$ in dimensionless units. Four different times are shown, where time is scaled by $a /|\boldsymbol{V}|$. The background porosity is 0.01 and is shown as white on the plots. Red indicates increased porosity from the background, blue shows reduced porosity. Note that a different colourscale is used in each time plot. $R=1000$. 

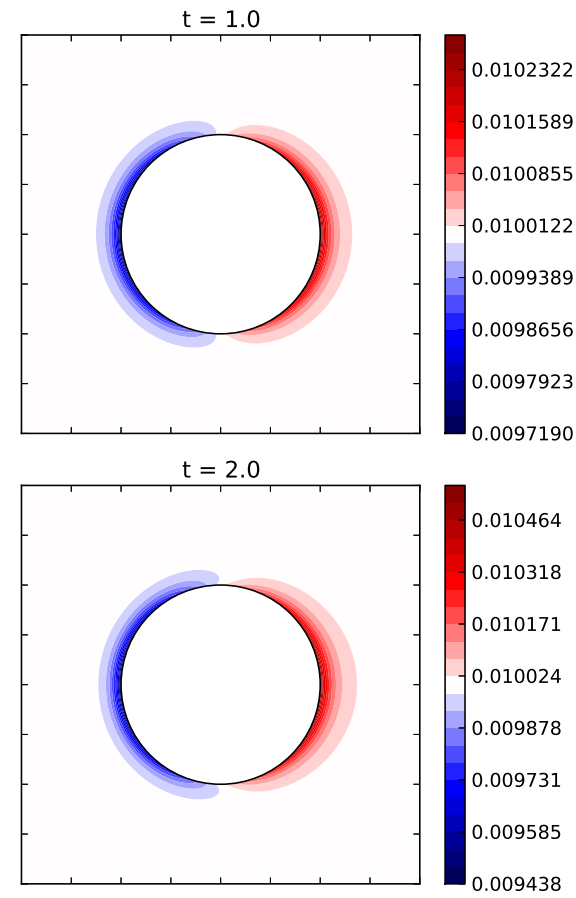

0.0097190

$t=3.0$

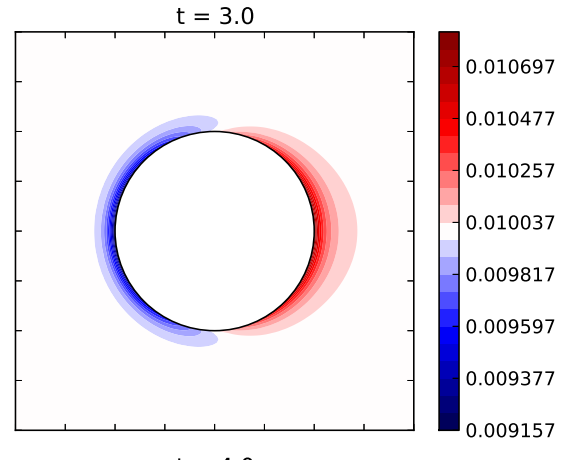

$\mathrm{t}=4.0$

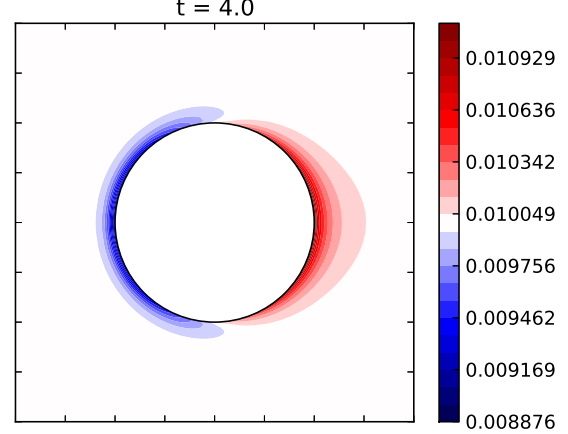

FIGURE 8. Plots showing porosity evolution around a translating sphere as in figure 7 except now the sphere has a dimensionless radius of 10.0. The background porosity and $R$ are unchanged from figure 7 . 

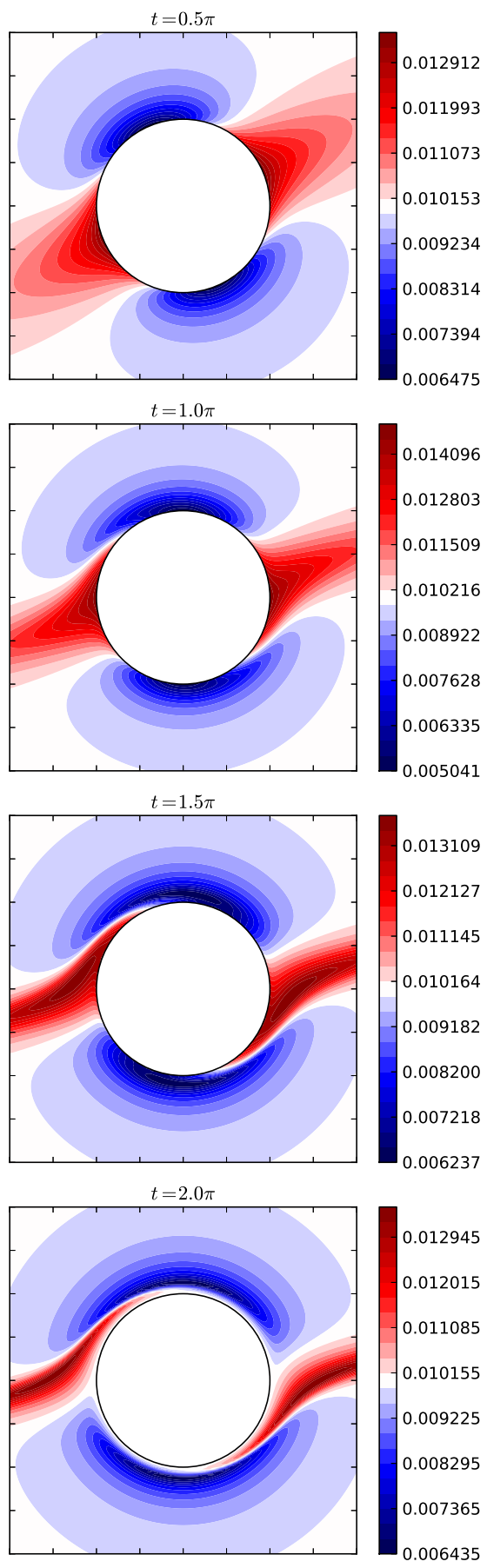

FIGURE 9. Plots showing the porosity evolution around a freely rotating sphere in a simple shear flow. The far field flow is the same as in figures 5 and 6 . The sphere has radius $a=0.001$ in dimensionless units. Four different times are shown, going up to a total strain of $2 \pi$, corresponding to a rotation of the sphere by $\pi$ radians. The background porosity is 0.01 and is shown as white on the plots. Red indicates increased porosity from the background, blue shows reduced porosity. Note that a different colourscale is used in each time plot. $R=1000$. 


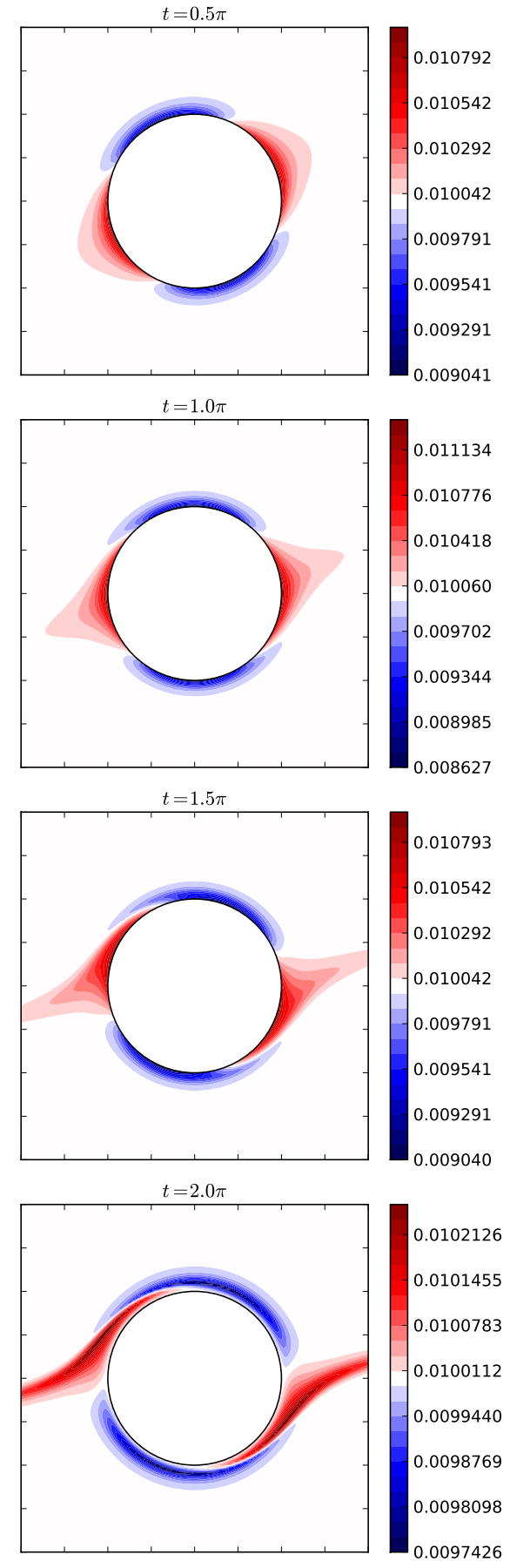

FIGURE 10. Plots showing the porosity evolution around a freely rotating sphere in a simple shear flow as in figure 9 except now the sphere has a dimensionless radius of 10.0. The background porosity and $R$ are unchanged from figure 9 . 
solution to the porosity evolution over time. To understand the time evolution fully requires a numerical solution of the compaction equations, work on which is currently ongoing using the finite element method (Alisic et al. 2013).

These analytical solutions have two immediate uses: First, they form a key benchmark for a full numerical solution to compare against. Second, they provide a prediction for the variation in porosity around a sphere in simple shear that can be compared with the results of laboratory deformation experiments (Qi et al. 2013). The model predicts a distinct asymmetry between the regions of high and low porosity that could be observed. The radial extent to which compaction extends may help to constrain the compaction length, and the ratio of bulk viscosity to shear viscosity may also be able to be constrained by the magnitude of the porosity variations observed after a given amount of strain. The comparison of the results of this study with laboratory experiments should form an excellent test of the ability of McKenzie's compaction equations to describe the deformation of partially molten rocks. Finally, these analytical solutions may prove useful in the interpretation of real rock microstructure (Manga 2005; McKenzie \& Holness 2000), the main motivation for McKenzie and Holness's original study of compacting flow past a sphere.

\section{Acknowledgements}

This work was support by NERC standard grant NE/I023929/1. I am grateful to David Kohlstedt and Chao Qi for bringing this problem to my attention. I would like to thank Richard Katz, Laura Alisic, and Garth Wells for a number of very useful discussions. I would like to thank three anonymous reviewers for their help in improving the clarity of this article. I would also like to thank John Rallison for his undergraduate lectures on Slow Viscous Flow, which provided much of the inspiration for the techniques used in this work.

\section{REFERENCES}

Acheson, D. J. 1990 Elementary Fluid Dynamics. Oxford University Press.

Alisic, L. , Rudge, J. F., Wells, G. N. , Katz, R. F. \& Rhebergen, S. 2013 Shear banding in a partially molten mantle. In SIAM Conference on Mathematical and Computational Issues in the Geosciences, Padova, Italy.

Batchelor, G. K. 1967 An Introduction to Fluid Dynamics. Cambridge University Press.

Griffiths, D. J. 1999 Introduction to Electrodynamics, 3rd edn. Prentice Hall.

Guazzelli, E. \& Morris, J. F. 2012 A Physical Introduction to Suspension Dynamics. Cambridge University Press.

Hewitt, I. J. \& Fowler, A. C. 2009 Melt channelization in ascending mantle. J. Geophys. Res. 114, B06210.

KAtz, R. F. 2010 Porosity-driven convection and asymmetry beneath midocean ridges. Geochem. Geophys. Geosyst. 11, Q0AC07.

Katz, R. F. , Spiegelman, M. \& Holtzman, B. 2006 The dynamics of melt and shear localization in partially molten aggregates. Nature 442, 676-679.

Kim, S. \& Karrila, S. J. 2005 Microhydrodynamics: Principles and Selected Applications. Dover.

Kohlstedt, D. L. \& Holtzman, B. K. 2009 Shearing melt out of the earth: An experimentalist's perspective on the influence of deformation on melt extraction. Annu. Rev. Earth Planet. Sci. 37, 561-593.

Lister, J. R., Kerr, R. C. , Russell, N. J. \& Crosby, A. 2011 Rayleigh-Taylor instability of an inclined buoyant viscous cylinder. J. Fluid Mech. 671, 313-338.

Manga, M. 2005 Deformation of flow bands by bubbles and crystals. Geological Society of America Special Papers 396, 47-53. 
McKenzIE, D. 1984 The generation and compaction of partially molten rock. J. Petrol. 25, 713-765.

McKenzie, D. \& Holness, M. 2000 Local deformation in compacting flows: development of pressure shadows. Earth Planet. Sci. Lett 180, 169-184.

Mittelstaedt, E. , Ito, G. \& van Hunen, J. 2011 Repeat ridge jumps associated with plumeridge interaction, melt transport, and ridge migration. J. Geophys. Res. 116, B01102.

NEubeR, H. 1934 Ein neuer ansatz zur lösung räumlicher probleme der elastizitätstheorie. Journal of Applied Mathematics and Mechanics 14, 203-212.

PApkovich, P. F. 1932 Solution générale des équations differentielles fondamentales d'élasticité exprimée par trois fonctions harmoniques. Compt. Rend. Acad. Sci. Paris 195, 513-515.

Phan-Thien, N. \& KIM, S. 1994 Microstructures in Elastic Media. Oxford University Press.

Poritsky, H. 1938 Generalizations of the gauss law of the spherical mean. Trans. Amer. Math. Soc. 43, 2 .

QI, C. , ZhaO, Y.-H. \& Kohlstedt, D. L. 2013 An experimental study of pressure shadows in partially molten rocks. Earth Planet. Sci. Lett 382, 77-84.

RicArd, Y. 2007 Physics of mantle convection. In Treatise on Geophysics (ed. Gerald Schubert), chap. 7.02 , p. 31-87. Elsevier.

Rudge, J. F., Bercovici, D. \& Spiegelman, M. 2011 Disequilibrium melting of a two phase multicomponent mantle. Geophys. J. Int. 184, 699-718.

Sabelfeld, K. K. \& Shalimova, I. A. 1997 Spherical means for PDEs. VSP.

Schiemenz, A. , Liang, Y. \& Parmentier, E. M. 2011 A high-order numerical study of reactive dissolution in an upwelling heterogeneous mantle-I. Channelization, channel lithology and channel geometry. Geophys. J. Int. 186, 641-664.

Simpson, G. , Spiegelman, M. \& Weinstein, M. I. 2010 A multiscale model of partial melts:1. Effective equations. J. Geophys. Res. 115, B04410.

Spiegelman, M. 1993 Flow in deformable porous media. Part 1 Simple analysis. J. Fluid Mech. 247, 17-38.

\section{Appendix A. A reciprocal theorem}

Suppose that $\left\{\tilde{\boldsymbol{v}}_{s}, \tilde{P}, \tilde{\boldsymbol{q}}\right\}$ and $\left\{\boldsymbol{v}_{s}^{\prime}, P^{\prime}, \boldsymbol{q}^{\prime}\right\}$ are both solutions to the compaction equations (2.2-2.5) on the same domain but with potentially different boundary conditions. From (2.4) we have that $\boldsymbol{v}_{s}^{\prime} \cdot(\boldsymbol{\nabla} \cdot \tilde{\bar{\sigma}})=0$, which when integrated over the domain $V$ yields

$$
\int_{S} \boldsymbol{v}_{s}^{\prime} \cdot \tilde{\bar{\sigma}} \cdot \boldsymbol{n} \mathrm{d} S=\int_{V} \tilde{\bar{\sigma}}: \boldsymbol{\nabla} \boldsymbol{v}_{s}^{\prime} \mathrm{d} V
$$

where $S$ is the surface bounding the domain. From (2.2) we have that $\tilde{P} \boldsymbol{\nabla} \cdot\left(\boldsymbol{v}_{s}^{\prime}+\boldsymbol{q}^{\prime}\right)=0$ which when integrated yields

$$
\int_{S} \tilde{P} \boldsymbol{q}^{\prime} \cdot \boldsymbol{n} \mathrm{d} S=\int_{V} \boldsymbol{q}^{\prime} \cdot \boldsymbol{\nabla} \tilde{P}-\tilde{P} \boldsymbol{\nabla} \cdot \boldsymbol{v}_{s}^{\prime} \mathrm{d} V .
$$

Subtraction of (A 2) from (A 1) and using (2.3) and (2.5) yields

$$
\int_{S}-\tilde{P} \boldsymbol{q}^{\prime} \cdot \boldsymbol{n}+\boldsymbol{v}_{s}^{\prime} \cdot \tilde{\bar{\sigma}} \cdot \boldsymbol{n} \mathrm{d} S=\int_{V} \mathcal{A}\left(\left\{\tilde{\boldsymbol{v}}_{s}, \tilde{P}, \tilde{\boldsymbol{q}}\right\},\left\{\boldsymbol{v}_{s}^{\prime}, P^{\prime}, \boldsymbol{q}^{\prime}\right\}\right) \mathrm{d} V .
$$

where $\mathcal{A}$ is the operator

$$
\mathcal{A}\left(\left\{\tilde{\boldsymbol{v}}_{s}, \tilde{P}, \tilde{\boldsymbol{q}}\right\},\left\{\boldsymbol{v}_{s}^{\prime}, P^{\prime}, \boldsymbol{q}^{\prime}\right\}\right) \equiv \frac{\mu}{\mathrm{k}_{\phi}} \tilde{\boldsymbol{q}} \cdot \boldsymbol{q}^{\prime}+\zeta_{\phi}\left(\boldsymbol{\nabla} \cdot \tilde{\boldsymbol{v}}_{s}\right)\left(\boldsymbol{\nabla} \cdot \boldsymbol{v}_{s}^{\prime}\right)+2 \eta_{\phi} \tilde{\epsilon}: \epsilon^{\prime}
$$

and $\epsilon$ is the trace-free part of the strain rate tensor,

$$
\epsilon \equiv \frac{1}{2}\left(\boldsymbol{\nabla} \boldsymbol{v}_{s}+\boldsymbol{\nabla} \boldsymbol{v}_{s}^{T}\right)-\frac{1}{3}\left(\boldsymbol{\nabla} \cdot \boldsymbol{v}_{s}\right) l .
$$


Since $\mathcal{A}$ is symmetric under the interchange of primes and tildes the following reciprocal theorem is obtained

$$
\int_{S}-\tilde{P} \boldsymbol{q}^{\prime} \cdot \boldsymbol{n}+\boldsymbol{v}_{s}^{\prime} \cdot \tilde{\bar{\sigma}} \cdot \boldsymbol{n} \mathrm{d} S=\int_{S}-P^{\prime} \tilde{\boldsymbol{q}} \cdot \boldsymbol{n}+\tilde{\boldsymbol{v}}_{s} \cdot \bar{\sigma}^{\prime} \cdot \boldsymbol{n} \mathrm{d} S .
$$

\section{Appendix B. Uniqueness}

When the solution with tildes and the solution with primes are identical, (A 3) becomes the equation of mechanical energy balance,

$$
\int_{S}-P \boldsymbol{q} \cdot \boldsymbol{n}+\boldsymbol{v}_{s} \cdot \bar{\sigma} \cdot \boldsymbol{n} \mathrm{d} S=\int_{V} \Psi \mathrm{d} V,
$$

where the left-hand side represents the rate of working at the boundaries and the righthand side represents the viscous dissipation. $\Psi$ is given by

$$
\Psi \equiv \frac{\mu}{\mathrm{k}_{\phi}} \boldsymbol{q} \cdot \boldsymbol{q}+\zeta_{\phi}\left(\boldsymbol{\nabla} \cdot \boldsymbol{v}_{s}\right)^{2}+2 \eta_{\phi} \epsilon: \epsilon .
$$

Suppose there exist two solutions to the compaction equations with identical boundary conditions on the normal component of Darcy flux $\boldsymbol{q} \cdot \boldsymbol{n}$ and the solid velocity $\boldsymbol{v}_{s}$. By linearity, the difference between these two solutions also satisfies the compaction equations, but with homogeneous boundary conditions, $\boldsymbol{q} \cdot \boldsymbol{n}=0, \boldsymbol{v}_{s}=\mathbf{0}$ on $S$. Homogeneous boundary conditions imply that the left-hand side of (B 1$)$ is zero, and hence that $\Psi=0$ everywhere in the domain (since $\Psi$ is positive semi-definite). $\Psi=0$ implies that

$$
\boldsymbol{q}=\mathbf{0}, \quad \boldsymbol{\nabla} \cdot \boldsymbol{v}_{s}=0, \quad \epsilon=0 .
$$

The only non-trivial solution of (B 3) is a rigid body rotation

$$
\boldsymbol{v}_{s}=\boldsymbol{V}+\boldsymbol{\Omega} \times \boldsymbol{x}, \quad P=\text { constant. }
$$

Since $\boldsymbol{v}_{s}$ satisfies homogeneous boundary conditions, it follows that $\boldsymbol{v}_{s}$ must be zero everywhere in the domain, and $P$ is a constant. Thus solutions to the compaction equations with boundary conditions on the normal component of Darcy flux $\boldsymbol{q} \cdot \boldsymbol{n}$ and the solid velocity $\boldsymbol{v}_{s}$ are unique up to at most a constant in the pressure. Other choices of boundary conditions (such as on tractions) which also make the left-hand side of (B 1) zero yield solutions which are unique up to at most a constant in the pressure and a rigid body motion.

\section{Appendix C. Cylindrical problems and other geometries}

The Papkovich-Neuber technique exploited throughout this manuscript can also be used to tackle problems in 2D, considering flows past circular cylinders rather than spheres. However, it is well-known that there is no bounded solution to the Stokes flow equations for uniform flow past a cylinder in an infinite domain. This result is known as the Stokes paradox, and as a consequence there is also no bounded solution to the compaction equations for uniform flow past a cylinder.

However, there does exist a finite solution to the problem of a circular cylinder placed in a straining flow, and it can be found by the same method as detailed for a sphere in section 5.3. In $2 \mathrm{D}$, the fundamental solution to Laplace's equation is $-(1 / 2 \pi) \log r$, and the fundamental solution to the modified Helmholtz equation is $(1 / 2 \pi) K_{0}(r)$ (where $K_{0}(r)$ is a modified Bessel function of the second kind). For a given strain rate tensor $E$, 
linearity suggests Papkovich-Neuber potentials of the form

$$
\begin{gathered}
\varphi=F E \cdot \nabla(\log r)=\frac{F E \cdot \boldsymbol{x}}{r^{2}}, \\
\chi=G E: \nabla \nabla(\log r)=\frac{-2 G \boldsymbol{x} \cdot E \cdot \boldsymbol{x}}{r^{4}}, \\
\mathcal{C}=H E: \nabla \nabla\left(K_{0}(r)\right)=\frac{H K_{2}(r) \boldsymbol{x} \cdot E \cdot \boldsymbol{x}}{r^{2}} .
\end{gathered}
$$

We could also have tried a potential of the form $E: \nabla \nabla \nabla(\log r)$ for $\varphi$ but this yields the same result as the $\chi$ does.

Substituting into (3.10) and (3.11) yields

$$
\begin{gathered}
\boldsymbol{v}_{s}=\left(-\frac{4 G}{r^{4}}+\frac{2 H K_{2}(r)}{r^{2}}\right) E \cdot \boldsymbol{x}+\left(-\frac{2 F}{r^{4}}+\frac{8 G}{r^{6}}-\frac{H K_{3}(r)}{r^{3}}\right)(\boldsymbol{x} \cdot E \cdot \boldsymbol{x}) \boldsymbol{x}, \\
P=\left(-\frac{4 \mathcal{B} F}{r^{4}}+\frac{H K_{2}(r)}{r^{2}}\right) \boldsymbol{x} \cdot E \cdot \boldsymbol{x} .
\end{gathered}
$$

Other quantities of interest are given by

$$
\begin{aligned}
\boldsymbol{\omega}_{s}= & \frac{4 F}{r^{4}} \boldsymbol{x} \times E \cdot \boldsymbol{x}, \\
\boldsymbol{q}= & \left(\frac{8 \mathcal{B} F}{r^{4}}-\frac{2 H K_{2}(r)}{r^{2}}\right) E \cdot \boldsymbol{x}+\left(-\frac{16 \mathcal{B} F}{r^{6}}+\frac{H K_{3}(r)}{r^{3}}\right)(\boldsymbol{x} \cdot E \cdot \boldsymbol{x}) \boldsymbol{x}, \\
e= & \left(-\frac{4 G}{r^{4}}+\frac{2 K_{2}(r) H}{r^{2}}\right) E \\
& +\left(-\frac{2 F}{r^{4}}+\frac{16 G}{r^{6}}-\frac{2 H K_{3}(r)}{r^{3}}\right)((E \cdot \boldsymbol{x}) \boldsymbol{x}+\boldsymbol{x}(E \cdot \boldsymbol{x})) \\
& +\left(-\frac{2 F}{r^{4}}+\frac{8 G}{r^{6}}-\frac{H K_{3}(r)}{r^{3}}\right)(\boldsymbol{x} \cdot E \cdot \boldsymbol{x}) \boldsymbol{I} \\
& +\left(\frac{8 F}{r^{6}}-\frac{48 G}{r^{8}}+\frac{H K_{4}(r)}{r^{4}}\right)(\boldsymbol{x} \cdot E \cdot \boldsymbol{x}) \boldsymbol{x} \boldsymbol{x}, \\
\bar{\sigma}= & 2 \mathcal{B}\left(\left(\frac{2 F}{r^{4}}-\frac{H K_{2}(r)}{r^{2}}\right)(\boldsymbol{x} \cdot E \cdot \boldsymbol{x}) \boldsymbol{I}+e\right) .
\end{aligned}
$$

The boundary conditions that $\boldsymbol{v}_{s}=-E \cdot \boldsymbol{x}$ and $\boldsymbol{q} \cdot \boldsymbol{n}=0$ on $r=a$ imply that

$$
\begin{gathered}
-\frac{4 G}{a^{4}}+\frac{2 H K_{2}(a)}{a^{2}}=-1, \\
-\frac{2 F}{a^{4}}+\frac{8 G}{a^{6}}-\frac{H K_{3}(a)}{a^{3}}=0, \\
-\frac{8 \mathcal{B} F}{a^{4}}-\frac{H K_{2}^{\prime}(a)}{a}=0,
\end{gathered}
$$

and thus

$$
\begin{gathered}
F=-\frac{a^{4} K_{2}^{\prime}(a)}{4 \mathcal{B} K_{1}(a)-a^{2} K_{2}^{\prime}(a)}, \\
G=\frac{a^{4}}{4}+\frac{4 a^{3} \mathcal{B} K_{2}(a)}{4 \mathcal{B} K_{1}(a)-a^{2} K_{2}^{\prime}(a)}, \\
H=\frac{8 a \mathcal{B}}{4 \mathcal{B} K_{1}(a)-a^{2} K_{2}^{\prime}(a)},
\end{gathered}
$$


which provides the solution. Adding back on the far-field velocity $E \cdot \boldsymbol{x}$, the traction around the cylinder is given by

$$
\left.\bar{\sigma} \cdot \boldsymbol{n}\right|_{r=a}=\frac{4 \mathcal{B} F}{a^{3}} E \cdot \boldsymbol{x},
$$

from which the stresslet can be calculated as

$$
S=4 \pi \mathcal{B} F E
$$

When $a \gg 1$

$$
S \sim 4 \pi \mathcal{B} a^{2} E
$$

which is the result for Stokes flow; and when $a \ll 1$,

$$
S \sim 4 \pi a^{2} \frac{\mathcal{B}}{1+\mathcal{B}} E
$$

which is the large compaction length limit and is smaller.

\section{C.1. Other geometries}

The Papkovich-Neuber technique can be generalised to a wide variety of different geometries. All that is needed are the appropriate solutions to Laplace's equation and the modified Helmholtz equation in that geometry. For example, solutions to the compaction equations within spheres and cylinders can found from the solutions to Laplace's equation that are bounded at the origin,

$$
r^{2 n+1} \nabla^{n} \frac{1}{r} \quad(3 \mathrm{D}), \quad r^{2 n} \nabla^{n} \log r \quad(2 \mathrm{D}),
$$

and the corresponding solutions of the modified Helmholtz equation

$$
\nabla^{n} i_{0}(r) \quad(3 \mathrm{D}), \quad \nabla^{n} I_{0}(r) \quad(2 \mathrm{D}),
$$

which are based on the modified Bessel functions of the first kind. 